\title{
Adaptation to the coupling of glycolysis to toxic methylglyoxal production in tpiA deletion strains of Escherichia coli requires synchronized and counterintuitive genetic changes
}

McCloskey, Douglas; Xu, Sibei; Sandberg, Troy E.; Brunk, Elizabeth; Hefner, Ying; Szubin, Richard; Feist, Adam M.; Palsson, Bernhard O.

Published in:

Metabolic Engineering

Link to article, DOI:

10.1016/j.ymben.2018.05.012

Publication date:

2018

Document Version

Peer reviewed version

Link back to DTU Orbit

Citation (APA):

McCloskey, D., Xu, S., Sandberg, T. E., Brunk, E., Hefner, Y., Szubin, R., Feist, A. M., \& Palsson, B. O. (2018). Adaptation to the coupling of glycolysis to toxic methylglyoxal production in tpiA deletion strains of Escherichia coli requires synchronized and counterintuitive genetic changes. Metabolic Engineering, 48, 82-93. https://doi.org/10.1016/j.ymben.2018.05.012

\section{General rights}

Copyright and moral rights for the publications made accessible in the public portal are retained by the authors and/or other copyright owners and it is a condition of accessing publications that users recognise and abide by the legal requirements associated with these rights.

- Users may download and print one copy of any publication from the public portal for the purpose of private study or research.

- You may not further distribute the material or use it for any profit-making activity or commercial gain

- You may freely distribute the URL identifying the publication in the public portal 


\section{Author's Accepted Manuscript}

Adaptation to the coupling of glycolysis to toxic methylglyoxal production in tpiA deletion strains of Escherichia coli requires synchronized and counterintuitive genetic changes

Douglas McCloskey, Sibei Xu, Troy E. Sandberg, Elizabeth Brunk, Ying Hefner, Richard Szubin, Adam M. Feist, Bernhard O. Palsson

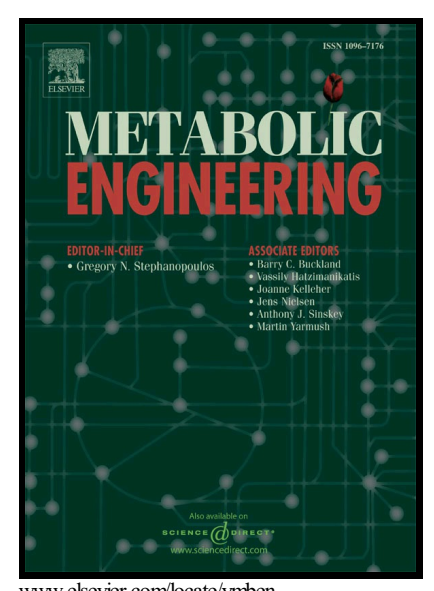

PII: $\quad$ S1096-7176(18)30063-6

DOI: $\quad$ https://doi.org/10.1016/j.ymben.2018.05.012

Reference: $\quad$ YMBEN1410

To appear in: Metabolic Engineering

Received date: 9 February 2018

Revised date: 3 May 2018

Accepted date: 23 May 2018

Cite this article as: Douglas McCloskey, Sibei Xu, Troy E. Sandberg, Elizabeth Brunk, Ying Hefner, Richard Szubin, Adam M. Feist and Bernhard O. Palsson, Adaptation to the coupling of glycolysis to toxic methylglyoxal production in tpiA deletion strains of Escherichia coli requires synchronized and counterintuitive genetic changes, Metabolic Engineering, https://doi.org/10.1016/j.ymben.2018.05.012

This is a PDF file of an unedited manuscript that has been accepted for publication. As a service to our customers we are providing this early version of the manuscript. The manuscript will undergo copyediting, typesetting, and review of the resulting galley proof before it is published in its final citable form. Please note that during the production process errors may be discovered which could affect the content, and all legal disclaimers that apply to the journal pertain. 
Adaptation to the coupling of glycolysis to toxic methylglyoxal production in tpiA deletion strains of Escherichia coli requires synchronized and counterintuitive genetic changes *

Douglas McCloskey ${ }^{1,2}$, Sibei Xu${ }^{1}$, Troy E. Sandberg ${ }^{1}$, Elizabeth Brunk ${ }^{1}$, Ying Hefner ${ }^{1}$, Richard Szubin $^{1}$, Adam M. Feist ${ }^{1,2}$, and Bernhard O. Palsson ${ }^{1,2, *}$

${ }^{1}$ Department of Bioengineering, University of California - San Diego, La Jolla, CA 92093, USA.

${ }^{2}$ Novo Nordisk Foundation Center for Biosustainability, Technical University of Denmark, 2800 Lyngby, Denmark.

${ }^{*}$ Corresponding author, Department of Bioengineering, University of California, San Diego, 9500 Gilman Drive, La Jolla, CA 92093-0412, USA. Tel.: 858-534-5668; Fax: 858-822-3120. palsson@ucsd.edu

\section{Abstract}

Methylglyoxal is a highly toxic metabolite that can be produced in all living organisms. Methylglyoxal was artificially elevated by removal of the tpiA gene from a growth optimized Escherichia coli strain. The initial response to elevated methylglyoxal and its toxicity was characterized, and detoxification mechanisms were studied using adaptive laboratory evolution. We found that: 1) Multi-omics analysis revealed biological consequences of methylglyoxal toxicity, which included attack on macromolecules including DNA and RNA and perturbation of nucleotide levels; 2) Counter-intuitive cross-talk between carbon starvation and inorganic phosphate signalling was revealed in the tpiA deletion strain that required mutations in inorganic phosphate signalling mechanisms to alleviate; and 3) The split flux through lower glycolysis depleted glycolytic intermediates requiring a host of synchronized and coordinated mutations in non-intuitive network locations in order to re-adjust the metabolic flux map to achieve optimal

\footnotetext{
Adaptive laboratory evolution, tpiA gene knockout, mutation analysis, multi-omics data integration, systems biology, E. coli
} 
growth. Such mutations included a systematic inactivation of the Phosphotransferase System (PTS) and alterations in cell wall biosynthesis enzyme activity. This study demonstrated that deletion of major metabolic genes followed by ALE was a productive approach to gain novel insight into the systems biology underlying optimal phenotypic states.

\section{Introduction}

Methylglyoxal (mthgxl) is a highly toxic metabolite that can be produced in all living organisms, and is directly linked to sugar metabolism. Methylglyoxal is highly cytotoxic owing to keto and aldehyde groups that can readily react with nearby metabolites and exposed units of macromolecules. For example, the keto and aldehyde groups can bind covalently to macromolecules such as DNA and proteins to form advanced glycation end products (AGEs) (Basta et al., 2004; Goldin et al., 2006; Singh et al., 2001; Thomas, 2011). Methylglyoxal is also mutagenic, and has been shown to interact with DNA through direct binding of guanine residues (Kasai et al., 1982; Murata-Kamiya et al., 1999). Due to the potential for methylglyoxal generation in all living organisms and its toxic effects to the cell, a greater understanding of its toxicity and mechanisms of detoxification are needed.

Methylglyoxal can be formed as a side product of glycolysis via the methylglyoxal pathway (Saadat and Harrison, 1999; Weber et al., 2005). Methylglyoxal levels can be artificially increased by bifurcating flux through lower glycolysis by removing the tpiA gene. tpiA encodes the Triosephosphate Isomerase (TPI) that is responsible for isomerizing dihydroxyacetone phosphate (dhap) to glyceraldehyde-3-phosphate (g3p). Loss of TPI forces dhap to be metabolized by the methylglyoxal pathway instead of the enzymes of lower glycolysis, which generates methylglyoxal. Removal of tpiA has been used in biotechnology applications to increase production of 1,2-propanediol, which has high commercial utility in the production of 
polymers (Shelley, 2007). Additional studies have also identified genes in potassium transport that allow for cells to better cope with exposure to methyglyxoal (Ozyamak et al., 2013). Further studies that both reveal genetic interventions for how to optimize flux through the methylglyoxal pathway and allow the cell to rapidly detoxify methylglyoxal are needed for efficient production of methylglyoxal derived compounds.

Flux through the methylglyoxal pathway for production of methylglyoxal derived compounds for biotechnology applications can be optimized by adaptive laboratory evolution (ALE) (Fong et al., 2006). ALE is an experimental approach that introduces a selection pressure (e.g., growth rate selection) in a controlled environmental setting (Dragosits and Mattanovich, 2013; Plucain et al., 2014; Tenaillon et al., 2016). Using ALE, organisms can be perturbed from their evolutionary optimized homeostatic states, and their re-adjustment to a new growth condition can be studied. Monitoring cellular changes during the course of adaptation can reveal novel and non-intuitive component functions and interactions that can then be re-engineered into a host organism. ALE of tpiA mutants found an increase in flux capacity through the methylglyoxal pathway (Fong et al., 2006), however, a systems level understanding of methylglyoxal detoxification as a result of mutations that appeared during ALE were not revealed.

In this study, the effects of methlyglyoxal toxicity on cellular functions were investigated by removing the tpiA gene from a growth optimized E. coli strain (LaCroix et al., 2015). The consequences of increased level of methylglyoxal was studied in the tpiA knock-out strains, that was then evolved to tolerance using ALE. Multi-omic data analysis revealed biochemical evidence of methylglyoxal toxicity and genetic changes that upregulated mechanisms to overcome its toxic effects. 


\section{Results}

\section{tpiA KO}

In order to eliminate the confounding variable of adaptation to the growth conditions of the experiment, a starting strain in which the gene $\mathrm{KO}$ was implemented was carefully chosen. A wild-type E. coli K-12 MG1655 strain previously evolved under glucose minimal media at $37^{\circ} \mathrm{C}$ (LaCroix et al., 2015) (denoted as "Ref") was selected in order to minimize biological changes caused by adaption to the loss of a gene product from those caused by adaption to the growth conditions of the experiment.

TPI (tpiA, Triosphosphate Isomerase) was removed from Ref to generate strain uTpiA (denoted "unevolved tpiA knockout strain"). The loss of tpiA resulted in an $80 \%$ loss in growth rate (Fig. 1D). Four UTpiA independently-inoculated starting cultures were simultaneously evolved on glucose minimal media at $37^{\circ} \mathrm{C}$ in an automated ALE platform (LaCroix et al., 2015; Sandberg et al., 2014) to generate endpoint strains, denoted "evolved tpiA knockout strains" or "eTpiA". A statistically significant increase in final fitness (Student's t-test, pvalue $<0.05$ ) was found in all ALE endpoints of the eTpiA lineages (ave \pm stdev $164 \pm 7 \%$ increase in fitness) compared to UTpiA.

\section{Loss of tpiA activated the glyoxylate pathways}

Fructose 1,6-bisphosphate (fdp) from upper glycolysis is split into dihydroxyacetone phosphate (dhap) and glyceraldehyde-3-phosphate (g3p) by fructose-bisphosphate aldolase (FBA) (Fig. 2A). g3p can be directly processed down the enzymatic machinery of lower glycolysis, but dhap must first be isomerized by Triosephosphate Isomerase (TPI) to g3p before glyceraldehyde 
phosphate dehydrogenase (GAPD) can catalyze the first step of lower glycolysis (Fig. 2A). Loss of enzymatic interconversion by TPI thus bifurcates glycolysis into lower glycolysis and pathways that degrade dhap, that involve methylglyoxal formation.

Reaction fluxes estimated from $\mathrm{C}^{13}$ labeling experiments and estimated using Genome-scale Metabolic Flux Analysis (MFA) (McCloskey et al., 2016a, 2016b) found that upper glycolytic flux was approximately evenly split between lower glycolysis and the methylglyoxal pathway in uTpiA (Fig 2B). LC-MS/MS-based metabolomics (McCloskey et al., 2015, 2014) found that bifurcation of lower glycolysis also led to an approximate $3.55 \log 2$ fold change buildup of dhap in uTpiA compared to Ref (Table S3). While not measured directly, LC-MS/MS analysis was able to measure the amounts of methylglyoxal break down products, which indicate an elevation in methylglyoxal (Fig. S2). An end product of the methylglyoxal pathway is D/L-Lactate (lac-DL), which can then be converted back to pyruvate (pyr), the end product of lower glycolysis, by lactate dehydrogenase (LDH) (Fig. 2C). Minimal amounts of intracellular lac-DL and no detectable lac-DL excretion coupled with overexpression of IIdD and $1.67 \mathrm{mmol}^{*} \mathrm{gDCW}-1^{*} \mathrm{hr}-1$ flux through LDH (approximately the same amount of flux through MGSA), indicated that the majority of lac-DL produced was converted back to pyr in uTpiA (Fig. 2D-E).

E. coli has four paths (Fig. 3A) to metabolize methylglyoxal: paths 1 and 2 lead to the production of propane 1,2-diol (12ppd-R), and paths 3 and 4 lead to the production of lac-DL. Unlike lac$\mathrm{DL}, E$. coli has no known enzyme to process $12 \mathrm{ppd}-\mathrm{R}$ and hence it can only be excreted out of the cell. RNA sequencing revealed that in UTpiA paths 1 and 2 were primarily activated with minor contribution from path 3 and no contribution from path 4 (Fig. 3B). In contrast, path 4 was highly activated in all eTpiA strains with varying degrees of path 2 and path 3 activation. Based on expression profiling and metabolomics measurements, it appears that the majority of dhap was metabolized to $12 p p d-R$ in UTpiA compared to lac-DL in eTpiA strains. 


\section{Known mechanisms of methylglyoxal toxicity}

Mechanisms of methylglyoxal toxicity are known and enable deeper interpretation of the multiomic data set generated (discussed in the sections below). We thus describe them briefly. Methylglyoxal is capable of attacking exposed nucleotides of single stranded RNA or DNA (Krymkiewicz, 1973), particularly at guanosine bases (Broude and Budowsky, 1971) (Fig. 4B). This toxicity has been shown to severely inhibit DNA replication through methylglyoxal cross links formed between guanosine residues of DNA and lysine and/or cysteine residues of the DNA polymerase (Murata-Kamiya and Kamiya, 2001). Methylglyoxal is also capable of oxidizing amino acids of RNA or DNA via Fenton's reaction (Imlay and Linn, 1988; Kang, 2003).

Methylglyoxal damage is not limited to nucleotides, but extends to proteins and peptides. The thiol of cysteine groups is susceptible to form methylglyoxal induced hemithioacetal adduct cross links with nearby peptide strands or glutathione (Ridderström and Mannervik, 2002) (Fig. 4C). Methylglyoxal can form lysine-lysine cross links via the Maillard Reaction (Nagaraj et al., 1996) (Fig. 4D). In addition to lysine, methylglyoxal has been shown to bind and modify arginine and cysteine residues (Lo et al., 1994). The glycation of lysine in particular has been shown to generate superoxide anions that are then capable of causing additional damage (Kang, 2003) (Fig. 4D). The mechanisms by which methylglyoxal damages proteins and peptides has been shown to be capable of inhibiting enzymatic function in vitro and in vivo (Kalapos, 1994).

ROS generated through Fenton Reactions involving methylglyoxal can lead to DNA strand breaks (Fig. 4E). 2-phosphoglycolyate is formed during repair of DNA strand breaks (Fig. 4F). 2-phosphoglycolyate can then be broken down by phosphoglycolate phosphatase (Gph) to 
glycolate (glyclt) and inorganic phosphate (pi). In addition, methylglyoxal forms abducts with dATP and dGTP (Frischmann et al., 2005; Papoulis et al., 1995).

\section{Elevated methylglyoxal induced global stress responses}

Global stress responders were activated in UTpiA (Fig. 4H). Expression of rpoS was significantly increased in UTpiA compared to Ref (Fig. 4H). rpoS encodes an alternative sigma factor to $r p o D$ that acts as a master regulator of general stress response that is itself induced during times of stress (see (Landini et al., 2014) for a review). Many universal response genes were also activated. $u s p B, u s p D, u s p F$, and $u s p G$ were all significantly upregulated in $u T p i A$ compared to Ref (Fig. 4H).

\section{Elevated methylglyoxal activated glycation responses}

Evidence for methylglyoxal damage to and glycation of DNA was revealed by expression profiling (Fig. 4H). Numerous chromosomal toxin-antitoxin (TA) pairs were found to be activated in UTpiA. Chromosomal TA systems respond to DNA damage and nutrient limitation by suppressing protein synthesis and promoting cell motility and biofilm formation (Magnuson, 2007; Ramisetty and Santhosh, 2017). Activation of chromosomal TA pairs have been shown to be associated with persister phenotypes (Kim and Wood, 2010; Luidalepp et al., 2011). TA pair $m q s R / m q s A$ was significantly upregulated in uTpiA compared to Ref (Fig. $4 \mathrm{H}$ ). mqsR inhibits protein synthesis by cleaving mRNA at GCU sites (Yamaguchi et al., 2009). Overexpression of $m q s R$ induces expression of $\operatorname{csp} D$, which was also significantly upregulated in UTpiA (Kim et al., 2010). cspD inhibits DNA replication and increases persister cell formation (Kim and Wood, 2010). TA pair yafQ/dinJ was significantly upregulated in UTpiA compared to Ref (Fig. $4 \mathrm{H}$ ). yafQ inhibits protein synthesis by interacting with the $50 \mathrm{~S}$ subunit of the ribosome where it cleaves mRNAs at AAA codons (Motiejūnaitè et al., 2007; Prysak et al., 2009). 


\section{Elevated methylglyoxal activated ROS responses}

Evidence for iron oxidation caused by ROS attack via Fenton's reaction on iron-sulfur clusters was found in the transcriptomics data. Evidence included shifts in the primary ribonucleotide reductases. E. coli has two ribonucleotide diphosphate reductases that are encoded by $\operatorname{nrd} A B$ and $n r d E F$ that operate under aerobic conditions and a ribonucleotide triphosphate reductase encoded by $n r d D G$ that operates under anaerobic conditions (Brown et al., 1969; Hantke, 1988). The ribonucleotide di-/tri-phosphate reductases act to generate deoxynucleotides for DNA and RNA synthesis (Torrents, 2014). Of the aerobic ribonucleotide reductases, nrdEF expression is induced under oxidative stress when iron is unavailable to activate $\operatorname{nrd} A B$ (Martin and Imlay, 2011; Monje-Casas et al., 2001). The entire nrdHIEF operon was significantly upregulated in uTpiA compared to Ref (and eTpiA strains) (Fig. 4H), while $\operatorname{nrdA}$ was significantly downregulated in UTpiA compared to Ref. Fur appeared to be activated based on expression profiles of the small regulatory RNA rhyB. rhyB was upregulated in uTpiA compared to Ref (and eTpiA strains).

The manganese importer encoded by $m n t H$ was upregulated in UTpiA compared to ref (and eTpiA strains) (Fig. $4 \mathrm{H}$ ). $m n t H$ is regulated by OxyR, Fur, and MntR, and its expression is induced under iron starvation (Anjem et al., 2009). All genes of the sufABCDSE operon except sufC and sufS were upregulated in UTpiA. compared to Ref (and eTpiA strains). The genes of the sUfABCDSE operon encodes enzymes that compose an alternative Fe-S cluster biogenesis pathways that is activated in times of oxidative stress and iron starvation (Lee et al., 2004; Outten et al., 2004). In contrast, the primary Fe-S cluster biogenesis pathway encoded by the ICS system was upregulated in eTpiA strains (Fig. 4H. Table S4) (Kim et al., 2014; Roche et al., 2015; Tokumoto et al., 2002). 


\section{Elevated methylglyoxal activated nucleotide synthesis responses}

Expression of nucleotide synthesis and salvage pathways were massively perturbed in uTpiA (Table S5, pvalue $<0.05$, topGO, ParentChild, Fischer). The majority of nucleotide synthesis and salvage pathways were significantly down-regulated in UTpiA compared to Ref (and eTpiA strains) (Table S4). Metabolomics also revealed massive perturbations in nucleotides, nucleosides, and nucleobases. In fact, almost all nucleotides, nucleosides, and nucleobases assayed were significantly changed in UTpiA compared to Ref (and eTpiA strains) (Fig. 4G, Table S3). For example, a significant drop in ATP (-0.68 log2 fold change compared to Ref) resulted in a significant drop in the Energy Charge ratio in UTpiA (from 0.90 in Ref to 0.82) (Table S3). In addition, nucleotide triphosphates GTP, ITP, UTP, dTTP, and dUTP were significantly depleted; nucleotide diphophosphates GDP, UDP, and dADP were significantly perturbed; nucleotide monophosphates CMP, dIMP, and dCMP were significantly changed; and nucleobases nucleotides Adenine, Guanosine, Hypoxanthine, Inosine, and Uridine were significantly perturbed in uTpiA compared to Ref (Fig. 4G, Table S3). These large changes in the nucleotide pools at all levels of phosphorylation could be indicative of perturbations of not just de novo synthesis and salvage, but also potentially break down from DNA, RNA, or direct attack by methylglyoxal.

Taken together, elevated methylglyoxal levels activated a number of stress responses that are consistent with reported detoxification responses. These responses were characterized in more detail here than in previous studies, and not only did they reveal mechanistic details and the global nature of the response, but their characterization provided the necessary starting point for the interpretation of the adjustments made during the ensuing ALE experiment. 


\section{ALE alleviated glyoxalase repression increasing flux through the}

\section{methylglyoxal pathway}

Path 4 relies on the Glyoxalase enzymes encoded by gloAB (Fig. 3A, Fig 5). Glyoxalase 1 binds methylglyoxal with Reduced Glutathione (gthrd) to form S-D-lactoyl-glutathione (lgt-S) (Thornalley, 2003) (Fig. 3A). Glyoxalase 2 then regenerates gthrd from lgt-S while enzymatically generating lac-D (Vander Jagt, 1993) (Fig. 3A). Expression of gloA is repressed by NemR (Fig. 5A-B); repression is enhanced by elevated levels of mthgxyl (Ozyamak et al., 2013; Umezawa et al., 2008) (Fig. 5C). A mutation in the nemR promoter region in eTpiA03, and mutations in the nemR small-molecule-binding domain or tetramerization regions in eTpiA01, 02, and 04 were found to offset NemR repression, and allowed for increased expression of gloA (Fig. 4A-D).

The increased expression of gloA appeared to have provided a fitness advantage by increasing the flux capacity of the methylglyoxal pathway (Fig. 2B). The enhanced conversion of dhap to lac-DL and subsequent conversion of lac-DL to pyr (Fig. 2E) substantially increased the levels of both lac-DL and pyr in eTpiA strains. The levels of lac-DL were 8.65, 8.37, 8.34, and 8.72 log2 fold change in eTpiA strains, respectively, compared to UTpiA, while levels of pyr were $10.39,10.12,10.08$, and $10.47 \log 2$ fold change in eTpiA strains, respectively, compared to uTpiA. In particular, the levels of pyr were severely depleted in UTpiA due to the forced bifurcation of flux that resulted from the loss of the TPI. The levels of pyr were $-4.51 \log 2$ fold change less in uTpiA compared to Ref. Unconverted lac-DL was excreted by the eTpiA strains (Fig. 1 D). In addition, the increased activity of the Glyxylase system also led also lead to an elevation in methyglyoxal derived detox products (Fig S2). 


\section{Transcription factor cross-talk induced expression of phosphate}

\section{starvation pathways}

Phosphate import in E. coli is tightly regulated by the PhoB/PhoR two component system (TCS) (Hsieh and Wanner, 2010) (Fig. 6A). Signalling by the PhoBR TCS involves the high affinity $A B C$ phosphate transporter encoded by the pstSCAB operon and a regulatory protein encoded by phoU (Aguena et al., 2002; Gardner et al., 2014) that coordinate phosphate import with phosphate metabolism (Fig. 6A). When extracellular phosphate levels are high, phosphorylation and activation of $\mathrm{PhoB}$ by $\mathrm{PhoR}$ is inhibited by PhoU; when extracellular phosphate levels are low, PhoR phosphorylates and activates PhoB (Fig. 6A). Phosphorylated PhoB then activates numerous operons believed to be involved in phosphate metabolism (Baek and Lee, 2007; Hengge, 2009; Yang et al., 2012). Among these is the phn operon that encodes genes required to metabolize methylphosphonate (Kamat et al., 2011; Metcalf and Wanner, 1993), which provides a source of phosphate under conditions of phosphate starvation (Metcalf and Wanner, 1993).

The majority of phoB controlled operons were upregulated in uTpiA compared to Ref (Fig. $6 \mathrm{H}$, Table S4). The genes of the phn operon were massively upregulated in UTpiA. A majority of other experimentally verified genes controlled by phoB were also significantly differentially expressed in UTpiA (Fig. 6H, Table S4). Given the tightly controlled amount of phosphate in the cultivation medium, lack of phosphate would seem improbable. However, cross-talk between other histidine kinases including $\mathrm{CreC}$ and $\mathrm{KpdD}$ have been experimentally verified (Wanner, 1993). Targets of CreB, the TF pair of the CreCB TCS, including all genes of the malEFG operon, recG, spoT, trmH, recG, and talA were all differentially expressed in UTpiA compared to Ref (Table S4). The only known target of $\mathrm{KpdE}$, the $k d p F A B C$ operon was not differentially 
expressed. KpdE is the TF of the KpdDE TCS. Most probable given the expression of PhoB, CreB, and KpdE gene targets was cross-talk between $\mathrm{CreC}$ and $\mathrm{PhoB}$. CreB is activated during the fermentation of glycolytic carbon sources that produce short chain organic acids such as 12ppd-R, lac-DL, and pyr (Cariss et al., 2008).

\section{The PhoBR two component system and PST Pi ABC transporter}

\section{were mutation hotspots during ALE}

A series of mutations were found in $p h o B, p h o R$, and $p s t A$ that altered expression of genes of the PhoB regulon (Fig. 6B-F). pstA was mutated in all eTpiA strains (Fig. 6F). The mutation truncated the transmembrane protein PstA from 286 to 38 residues. Given that the PstA component is an integral part of the PST ABC transporter, it would seem likely that the truncation would inhibit the ability to transport phosphate, and would disrupt normal complex formation. The PST ABC transporter is the primary phosphate transporter in E. coli (Beard et al., 2000; Harris et al., 2001; Jackson et al., 2008). Loss of any of the PST components has been shown to change interaction of PstB with PhoU, leading to changes in phosphorylation patterns of PhoB by PhoR (Chekabab et al., 2014). Deletion of the PST system has been associated with virulence in Extraintestinal pathogenic E. coli (ExPEC) (Crépin et al., 2012; Daigle et al., 1995; Glattard et al., 2010; Lamarche and Harel, 2010).

Mutations were found in either phoB or phoR that fixed in eTpiA02, 03, and 04 strains, while lower frequency mutations were found in $p h o B$ and phoR in eTpiA01 (Fig. 6B-D). These mutations targeted the PhoB TF binding site of the phoBR operon (Fig. 6B), altered the amino acid sequence of PhoB (Fig. 6C), shortened the length of the PhoR peptide (Fig. 6D), or hit the phoB response regulatory domain (Fig. $6 \mathrm{E}$ ). The mutations in eTpiA02 and 03 resulted in the most deactivation of PhoB regulation, while the mutations in eTpiA01 and eTpiA04 retained 
similar expression profiles to that of uTpiA (Fig. $6 \mathrm{H}$ ). Interestingly, expression patterns of CreB targets in eTpiA01 and eTpiA04 also showed similar expression profiles to that of UTpiA indicating continued cross-talk with CreC (Table S4).

\section{Bifurcated glycolytic flux perturbed energy and redox carrier}

\section{balances, which lead to a global rerouting in the flux map to}

\section{regain optimal growth}

While lower glycolysis generates four energy equivalents (i.e., ATP) and two redox equivalents (i.e., NADH), the methylglyoxal pathway generates a toxic intermediate (i.e., methylglyoxal) and burns zero to two redox equivalents (i.e., NADH, NADPH, Reduced Glutathione) depending upon the pathway used. Consequently, levels of ATP were significantly depleted in UTpiA (-0.74 log2 fold change compared to Ref), which lead to a significant drop in the energy charge ratio (from 0.90 in Ref to 0.82 ) (Table S3). In addition, lower glycolytic intermediates including 1-3/23 diphosphoglycerate (13/23dpg), 2/3-phosphoglycerate, (2/3pg), pep, and pyr were severely depleted in UTpiA (-1.39, $-2.62,-1.47$, and -4.51 log2 fold change compared to Ref) (Table S3).

In particular, the depleted levels of pep appeared to be most detrimental to growth, and required major adjustments to all reactions and subsystems that involved pep. These included mutations to the Phosphotransferase System (PTS) and lower glycolytic enzymes. Major adjustments in flux also extended to subsystems that do not directly involve pep, but contributed flux to or from the pep node. These included upper glycolysis, the pentose phosphate pathway (PPP), and the TCA cycle. These adjust are detailed below. 


\section{Systematic decoupling of pep production from glucose import and}

\section{cell wall recycling occurred during ALE}

A host of mutations involving ptsG, galR, and nagA, nagC, and nagE in the eTpiA strains were found that appeared to systematically decouple pep consumption from carbon import. Mutations that altered the expression and function of nAcGam import and catabolic genes were found in eTpiA02/04 strains (Fig. 7). The nagBAC and nagE operons are negatively regulated by the NagC TF (Fig. 7A). EIIB encoded by nagE of the nAcGam PTS Permease imports and phosphorylates periplasmic $\mathrm{N}$-acetyl glucosamine (Fig. 7B). The phosphate is derived from the conversion of phosphoenolpyruvate (pep) to pyruvate (pyr) after a series of phosphorylation reactions along the PTS components El, HPr, and EIIA. N-acetyl glucosamine 6-phosphate (acgam6p) is then broken down to nitrate (nh4) and fructose-6-phosphate (f6p) by Nacetylglucosamine-6-phosphate deacetylase (AGDC) encoded by nagA and Glucosamine-6phosphate deaminase (G6PDA) encoded by nagB (Fig. 7B). The genes that compose the nagBAC and nagE operons contribute to a significant recycling of peptidoglycan (Plumbridge, 2009). All nagC mutations in eTpiA02/04 appeared to be deleterious resulting in truncated peptides (Fig. 7C). The nagC mutations relieved NagC repression of the nagBAC and nagE operons, resulting in significantly higher expression in eTpiA02/04 (Fig. 7D). Increased expression appeared to be offset by mutations that prevent import and phosphorylation of acgam by nagE. The nagE mutations occur in the PTSEIIC type-1 complex formation region and resulted in amino acid changes that could have affected activity (Table S8).

Mutations that increased expression of non-PTS glucose importers occurred in eTpiA01/03 strains (Fig. 8). The primary importer of glucose in E. coli is the PTS, which is similar to the nAcGam PTS imports and phosphorylates glucose at the expense of pep (Fig. 8A). Specifically, 
glucose is imported and phosphorylated from the periplasm into the cytosol by EllB encoded by ptsG. Alternative passive (i.e., D-galactose symporter encoded by galP) and active importers (i.e., D-galactose $A B C$ transporter encoded by $m g / B A C$ ) utilize the proton gradient or ATP, respectively, to import glucose (Fig. 8B). Passive and active importers are regulated by the GalR and GalS TFs (Fig. 8C). Mutations in galR in eTpiA01/03 appeared to alter activity so as to increase expression of galETKM and passive importer encoded by galP, but not active ATPdependent importer encoded by mgIBAC (Fig. 8F). In addition, a galP INS mutation occurred at the NagC binding site in eTpiA01, further indicating an adaptive preference for passive import overactive import (Fig. 8D). Note that eTpiA01 had the highest level of expression of galP.

Complementary mutations in eTpiA01/03 occurred in ptsG in that appeared to abolish import and phosphorylation activity of the primary PTS-dependent glucose importer. The ptsG SNP mutation in eTpiA01 introduced a stop codon that truncates the cytosolic portion of the protein including the PTS EIIB type-1 active site and phosphocysteine residue (Fig. 8E). The ptsG INS mutation (not shown in the figure) introduced an additional codon that shifted all residues by one in the PTS EIIB active site, which could have altered activity (Table S8).

\section{Additional systematic decoupling of pep enzymatic reactions}

In addition to carbon import processes, other enzymatic reactions that consume pep were also targeted by mutations. Mutations were found in pykF and murA that potentially affected enzymatic function (Fig. 9). Pyruvate kinase (PYK) is encoded by pykF or pykA and converts pep to pyr while generating ATP (Fig. 9A). Mutations found in pykF in eTpiA02, 03, and 04 were most likely deleterious (Fig. 9B). The pykF MOB resulted in a truncated peptide that removed all features except for two potassium ion binding sites and a substrate binding residue. The pykF DEL was located at the interface between the dimers, and resulted in a frameshift that 
shifted the stop codon beyond the normal length of the peptide that most likely generated an aggregate peptide. The pykF SNP changed the 226 residue from $G$ to $D$, and was located in close proximity to the magnesium binding site residues. UDP-N-acetylglucosamine 1carboxyvinyltransferase (UAGCVT), encoded by murA, converts UDP-N-acetyl-a-Dglucosamine (uacgam) to UDP-N-acetyl-a-D-glucosamine-enolpyruvate (uaccg) at the expense of pep in the first committed step to peptidoglycan biosynthesis (Fig. 9C). The murA DEL in eTpiA04 resulted in a non-frameshift that shortened the peptide by one amino acid (Fig. 9D). Interestingly, the mutation changed the cysteine residue at position 115 , which interacts with pep. The 115 residue has been actively investigated for implications in drug discovery (Bachelier et al., 2006; Barbosa et al., 2002; Baum et al., 2001; Couce et al., 2012; Desai and Miller, 2010; Dunsmore et al., 2008; Francisco et al., 2004; Kim et al., 1996; Miller et al., 2010; Xu et al., 2006).

\section{Multiple pathway flux adjustments during ALE balanced methylglyoxal generation with biosynthesis}

Bifurcation of lower glycolysis coupled glucose metabolism to the generation of the toxic intermediate methylglyoxal. A complete rewiring of metabolic flux through central metabolism was required for the eTpiA strains to recover approximately $50 \%$ of the growth rate of Ref. Notable shifts in flux were found in the pentose phosphate pathway (PPP), lower glycolysis, flux into the TCA cycle either through the anaeplerotic reaction Phosphoenol Pyruvate Carboxylase (PPC) or Citrate Synthase (CS), and utilization of the glyoxylate shunt. A 5.14 log2 fold change in flux per mol glucose through the Entner-Doudoroff (ED) pathway compared to Ref in UTpiA shifted to $3.60,2.46,3.36$, and $4.76 \log 2$ fold change in eTpiA strains, respectively. A -1.69 log2 fold change in flux per mol glucose through PYK compared to Ref in UTpiA shifted to -5.94, $-5.49,-6.36$, and $-4.49 \log 2$ fold change in eTpiA strains, respectively. The relative drop in PYK 
flux in many of the endpoints could in part be attributed decreased expression, but also due to mutations found in the pykF gene (discussed previously). The majority of pyr levels were recovered through the rejoining of flux through the methylglyoxal pathway instead of through PTS reactions and PYK. A 1.7 mmol*gDCW-1*hr-1 flux in from lac-DL to pyr in uTpiA increased to $4.9,6.2,4.7$, and 2.5 in eTpiA strains, respectively.

Overall flux into the TCA cycle per mol of glucose dropped in all eTpiA strains. An initial $90 \%$ relative boost per mole of glucose in PPC flux compared to Ref in UTpiA was lost to a -54\%, $15 \%,-49 \%$, and $-31 \%$ relative drop in eTpiA strains, respectively. An initial $182 \%$ relative boost per mole of glucose in CS flux compared to Ref in UTpiA was reduced to $17 \%, 96 \%, 34 \%$, and $90 \%$ in eTpiA strains, respectively. In addition, partial utilization of the glyoxylate shunt was restored in eTpiA strains. A $-94 \%$ relative change per mole of glucose through the glyoxylate shunt compared to Ref in UTpiA was increased a to $57 \%, 58 \%, 103 \%$, and $52 \%$ in eTpiA strains, respectively.

The above shifts in flux appeared to simultaneously increase flux through lower glycolysis (i.e., increased flux through ED) to increase the levels of lower glycolytic intermediates and generation of ATP and redox equivalents while minimizing glycolytic flux that would commit 1 mol of glucose to methylglyoxal generation. In addition, the above shifts in flux also maximized pyr production while minimizing pep consumption resulting in an overall loss in flux through the TCA cycle. Interestingly, while many of the redox ratios were recovered, the energy charge ratio was never recovered (Table S3), indicating that the coupling of biomass production to methylglyoxal production induced an intrinsic cost to energy production. 


\section{Conclusion}

Flux through glycolysis was stoichiometrically coupled to the production of the toxic metabolite methylglyoxal in $E$. coli by removing the tpiA gene. Production of methylglyoxal resulted in cellular damage that was overcome through efficient detoxification by the Glyoxalase enzymes. Elevation in the end-products of the methylglyoxal pathway, 12ppd-R and lac-DL, triggered an erroneous phosphate starvation response via cross talk with CreCB TCS that was dampened through mutations that affect $\mathrm{PhoB}$ signalling. Split flux through lower glycolysis depleted glycolytic intermediates, in particular, pep. Pep levels were restored to levels similar to Ref through mutations that decoupled pep production from carbon import. The detoxification strategy of methylglyoxal and network changes required an increase in carbon import without exacerbating methylglyoxal toxicity or depletion of glycolytic intermediates that serve as key biomass precursors. Thus, multi-omics analysis and ALE confirmed in higher resolution than previously reported methylglyoxal toxicity responses and revealed novel biochemical mechanisms that should provide researchers with valuable insights into strategies deployed for metabolic detoxification in bacteria.

Taken together, this study shows that the combination of KO, multi-omic data analysis and ALE represent a new line of biological inquiry. It reveals the full consequences of the loss of a gene product and the genetic changes needed to regain fitness. This overall conclusion provides an impetus to perform analogous studies for a series of major gene products in E. coli. Such studies would provide us with a new and deep understanding of the systems biology of basic regulatory and metabolic processes in bacteria. 


\section{Contributions}

D.M. designed the experiments; generated the strains; conducted all aspects of the metabolomics, fluxomics, phenomics, transcriptomics, and genomics experiments; performed all multi-omics statistical, graph, and modeling analyses; and wrote the manuscript. T.E.S. ran the ALE experiments. E.B. assisted with structural analysis. R.S. processed the DNA and RNA samples. S.X. assisted with metabolomics and fluxomics data collection, sample processing, and peak integration. Y.H. assisted with fluxomics data collection and sample processing. A.M.F designed and supervised the evolution experiments, and contributed to the data analysis and the manuscript. B.O.P conceived and outlined the study, supervised the data analysis, and co-wrote the manuscript.

\section{Acknowledgements}

We thank José Utrilla for helpful discussion and guidance when implementing the knockouts in the pre-evolved strain. We thank Jamey Young for helpful discussions throughout the MFA analysis. We thank Laurence Yang for helpful discussions regarding optimization and statistical analysis. This work was funded by the Novo Nordisk Foundation Grant Number NNF10CC1016517.

\section{Competing financial interests}

The authors declare no competing financial interests. 


\section{References}

Aguena, M., Yagil, E., Spira, B., 2002. Transcriptional analysis of the pst operon of Escherichia coli. Mol. Genet. Genomics 268, 518-524.

Anjem, A., Varghese, S., Imlay, J.A., 2009. Manganese import is a key element of the OxyR response to hydrogen peroxide in Escherichia coli. Mol. Microbiol. 72, 844-858.

Bachelier, A., Mayer, R., Klein, C.D., 2006. Sesquiterpene lactones are potent and irreversible inhibitors of the antibacterial target enzyme MurA. Bioorg. Med. Chem. Lett. 16, 56055609.

Baek, J.H., Lee, S.Y., 2007. Transcriptome analysis of phosphate starvation response in Escherichia coli. J. Microbiol. Biotechnol. 17, 244-252.

Barbosa, M.D.F.S., Yang, G., Fang, J., Kurilla, M.G., Pompliano, D.L., 2002. Development of a whole-cell assay for peptidoglycan biosynthesis inhibitors. Antimicrob. Agents Chemother. 46, 943-946.

Basta, G., Schmidt, A.M., De Caterina, R., 2004. Advanced glycation end products and vascular inflammation: implications for accelerated atherosclerosis in diabetes. Cardiovasc. Res. 63, 582-592.

Baum, E.Z., Montenegro, D.A., Licata, L., Turchi, I., Webb, G.C., Foleno, B.D., Bush, K., 2001. Identification and characterization of new inhibitors of the Escherichia coli MurA enzyme. Antimicrob. Agents Chemother. 45, 3182-3188.

Beard, S.J., Hashim, R., Wu, G., Binet, M.R., Hughes, M.N., Poole, R.K., 2000. Evidence for the transport of zinc(II) ions via the pit inorganic phosphate transport system in Escherichia coli. FEMS Microbiol. Lett. 184, 231-235.

Broude, N.E., Budowsky, E.I., 1971. The reaction of glyoxal with nucleic acid components III. Kinetics of the reaction with monomers. Biochimica et Biophysica Acta (BBA) - Nucleic Acids and Protein Synthesis 254, 380-388.

Brown, N.C., Canellakis, Z.N., Lundin, B., Reichard, P., Thelander, L., 1969. Ribonucleoside diphosphate reductase. Purification of the two subunits, proteins B1 and B2. Eur. J. Biochem. 9, 561-573.

Cadet, J., Douki, T., Gasparutto, D., Ravanat, J.-L., 2003. Oxidative damage to DNA: formation, measurement and biochemical features. Mutat. Res. 531, 5-23.

Cariss, S.J.L., Tayler, A.E., Avison, M.B., 2008. Defining the growth conditions and promoterproximal DNA sequences required for activation of gene expression by $\mathrm{CreBC}$ in Escherichia coli. J. Bacteriol. 190, 3930-3939.

Chekabab, S.M., Harel, J., Dozois, C.M., 2014. Interplay between genetic regulation of phosphate homeostasis and bacterial virulence. Virulence 5, 786-793.

Cooke, M.S., Evans, M.D., Dizdaroglu, M., Lunec, J., 2003. Oxidative DNA damage: mechanisms, mutation, and disease. FASEB J. 17, 1195-1214.

Couce, A., Briales, A., Rodríguez-Rojas, A., Costas, C., Pascual, A., Blázquez, J., 2012. Genomewide overexpression screen for fosfomycin resistance in Escherichia coli: MurA confers clinical resistance at low fitness cost. Antimicrob. Agents Chemother. 56, 27672769.

Crépin, S., Houle, S., Charbonneau, M.-È., Mourez, M., Harel, J., Dozois, C.M., 2012. Decreased expression of type 1 fimbriae by a pst mutant of uropathogenic Escherichia coli reduces urinary tract infection. Infect. Immun. 80, 2802-2815.

Daigle, F., Fairbrother, J.M., Harel, J., 1995. Identification of a mutation in the pst-phoU operon that reduces pathogenicity of an Escherichia coli strain causing septicemia in pigs. Infect. Immun. 63, 4924-4927.

Desai, K.K., Miller, B.G., 2010. Recruitment of genes and enzymes conferring resistance to the nonnatural toxin bromoacetate. Proc. Natl. Acad. Sci. U. S. A. 107, 17968-17973. 
Dragosits, M., Mattanovich, D., 2013. Adaptive laboratory evolution -- principles and applications for biotechnology. Microb. Cell Fact. 12, 64.

Dunsmore, C.J., Miller, K., Blake, K.L., Patching, S.G., Henderson, P.J.F., Garnett, J.A., Stubbings, W.J., Phillips, S.E.V., Palestrant, D.J., Angeles, J.D.L., Leeds, J.A., Chopra, I., Fishwick, C.W.G., 2008. 2-Aminotetralones: novel inhibitors of MurA and MurZ. Bioorg. Med. Chem. Lett. 18, 1730-1734.

Eschenburg, S., Schonbrunn, E., 2004. C115S MurA liganded with reaction products. https://doi.org/10.2210/pdb1 ryw/pdb

Fong, S.S., Nanchen, A., Palsson, B.O., Sauer, U., 2006. Latent pathway activation and increased pathway capacity enable Escherichia coli adaptation to loss of key metabolic enzymes. J. Biol. Chem. 281, 8024-8033.

Francisco, G.D., Li, Z., Albright, J.D., Eudy, N.H., Katz, A.H., Petersen, P.J., Labthavikul, P., Singh, G., Yang, Y., Rasmussen, B.A., Lin, Y.-I., Mansour, T.S., 2004. Phenyl thiazolyl urea and carbamate derivatives as new inhibitors of bacterial cell-wall biosynthesis. Bioorg. Med. Chem. Lett. 14, 235-238.

Frischmann, M., Bidmon, C., Angerer, J., Pischetsrieder, M., 2005. Identification of DNA adducts of methylglyoxal. Chem. Res. Toxicol. 18, 1586-1592.

Gardner, S.G., Johns, K.D., Tanner, R., McCleary, W.R., 2014. The PhoU protein from Escherichia coli interacts with PhoR, PstB, and metals to form a phosphate-signaling complex at the membrane. J. Bacteriol. 196, 1741-1752.

Glattard, E., Welters, I.D., Lavaux, T., Muller, A.H., Laux, A., Zhang, D., Schmidt, A.R., Delalande, F., Laventie, B.-J., Dirrig-Grosch, S., Colin, D.A., Van Dorsselaer, A., Aunis, D., Metz-Boutigue, M.-H., Schneider, F., Goumon, Y., 2010. Endogenous morphine levels are increased in sepsis: a partial implication of neutrophils. PLoS One 5, e8791.

Goldin, A., Beckman, J.A., Schmidt, A.M., Creager, M.A., 2006. Advanced glycation end products: sparking the development of diabetic vascular injury. Circulation 114, 597-605.

Gray, M.J., Li, Y., Leichert, L.I.-O., Xu, Z., Jakob, U., 2015. Does the Transcription Factor NemR Use a Regulatory Sulfenamide Bond to Sense Bleach? Antioxid. Redox Signal. 23, 747754.

Hantke, K., 1988. Characterization of an iron sensitive Mud1 mutant in E. coli lacking the ribonucleotide reductase subunit B2. Arch. Microbiol. 149, 344-349.

Harris, R.M., Webb, D.C., Howitt, S.M., Cox, G.B., 2001. Characterization of PitA and PitB from Escherichia coli. J. Bacteriol. 183, 5008-5014.

Hengge, R., 2009. Principles of C-di-GMP signalling in bacteria. Nat. Rev. Microbiol. 7, 263-273.

Hsieh, Y.-J., Wanner, B.L., 2010. Global regulation by the seven-component Pi signaling system. Curr. Opin. Microbiol. 13, 198-203.

Imlay, J.A., Linn, S., 1988. DNA damage and oxygen radical toxicity. Science 240, 1302-1309.

Jackson, R.J., Binet, M.R.B., Lee, L.J., Ma, R., Graham, A.I., McLeod, C.W., Poole, R.K., 2008. Expression of the PitA phosphate/metal transporter of Escherichia coli is responsive to zinc and inorganic phosphate levels. FEMS Microbiol. Lett. 289, 219-224.

Kalapos, M.P., 1994. Methylglyoxal toxicity in mammals. Toxicol. Lett. 73, 3-24.

Kamat, S.S., Williams, H.J., Raushel, F.M., 2011. Intermediates in the transformation of phosphonates to phosphate by bacteria. Nature 480, 570-573.

Kang, J.H., 2003. Oxidative damage of DNA by the reaction of amino acid with methylglyoxal in the presence of Fe (III). Int. J. Biol. Macromol. 33, 43-48.

Kasai, H., Kumeno, K., Yamaizumi, Z., Nishimura, S., Nagao, M., Fujita, Y., Sugimura, T., Nukaya, H., Kosuge, T., 1982. Mutagenicity of methylglyoxal in coffee. Gan 73, 681-683.

Kim, D.H., Lees, W.J., Kempsell, K.E., Lane, W.S., Duncan, K., Walsh, C.T., 1996. Characterization of a Cys115 to Asp substitution in the Escherichia coli cell wall biosynthetic enzyme UDP-GIcNAc enolpyruvyl transferase (MurA) that confers resistance to inactivation by the antibiotic fosfomycin. Biochemistry 35, 4923-4928. 
Kim, J.H., Bothe, J.R., Frederick, R.O., Holder, J.C., Markley, J.L., 2014. Role of IscX in ironsulfur cluster biogenesis in Escherichia coli. J. Am. Chem. Soc. 136, 7933-7942.

Kim, Y., Wang, X., Zhang, X.-S., Grigoriu, S., Page, R., Peti, W., Wood, T.K., 2010. Escherichia coli toxin/antitoxin pair MqsR/MqsA regulate toxin CspD. Environ. Microbiol. 12, 11051121.

Kim, Y., Wood, T.K., 2010. Toxins Hha and CspD and small RNA regulator Hfq are involved in persister cell formation through MqsR in Escherichia coli. Biochem. Biophys. Res. Commun. 391, 209-213.

Krymkiewicz, N., 1973. Reactions of methylglyoxal with nucleic acids. FEBS Lett. 29, 51-54.

LaCroix, R.A., Sandberg, T.E., O'Brien, E.J., Utrilla, J., Ebrahim, A., Guzman, G.I., Szubin, R., Palsson, B.O., Feist, A.M., 2015. Use of Adaptive Laboratory Evolution To Discover Key Mutations Enabling Rapid Growth of Escherichia coli K-12 MG1655 on Glucose Minimal Medium. Appl. Environ. Microbiol. 81, 17-30.

Lamarche, M.G., Harel, J., 2010. Membrane homeostasis requires intact pst in extraintestinal pathogenic Escherichia coli. Curr. Microbiol. 60, 356-359.

Landini, P., Egli, T., Wolf, J., Lacour, S., 2014. sigmaS, a major player in the response to environmental stresses in Escherichia coli: role, regulation and mechanisms of promoter recognition. Environ. Microbiol. Rep. 6, 1-13.

Lee, J.-H., Yeo, W.-S., Roe, J.-H., 2004. Induction of the sufA operon encoding Fe-S assembly proteins by superoxide generators and hydrogen peroxide: involvement of OxyR, IHF and an unidentified oxidant-responsive factor. Mol. Microbiol. 51, 1745-1755.

Lo, T.W., Westwood, M.E., McLellan, A.C., Selwood, T., Thornalley, P.J., 1994. Binding and modification of proteins by methylglyoxal under physiological conditions. A kinetic and mechanistic study with $\mathrm{N}$ alpha-acetylarginine, $\mathrm{N}$ alpha-acetylcysteine, and $\mathrm{N}$ alphaacetyllysine, and bovine serum albumin. J. Biol. Chem. 269, 32299-32305.

Luidalepp, H., Jõers, A., Kaldalu, N., Tenson, T., 2011. Age of inoculum strongly influences persister frequency and can mask effects of mutations implicated in altered persistence. J. Bacteriol. 193, 3598-3605.

Magnuson, R.D., 2007. Hypothetical functions of toxin-antitoxin systems. J. Bacteriol. 189, 6089-6092.

Martin, J.E., Imlay, J.A., 2011. The alternative aerobic ribonucleotide reductase of Escherichia coli, NrdEF, is a manganese-dependent enzyme that enables cell replication during periods of iron starvation. Mol. Microbiol. 80, 319-334.

McCloskey, D., Gangoiti, J.A., Palsson, B.O., Feist, A.M., 2015. A pH and solvent optimized reverse-phase ion-paring-LC-MS/MS method that leverages multiple scan-types for targeted absolute quantification of intracellular metabolites. Metabolomics 11, 1338-1350.

McCloskey, D., Utrilla, J., Naviaux, R.K., Palsson, B.O., Feist, A.M., 2014. Fast Swinnex filtration (FSF): a fast and robust sampling and extraction method suitable for metabolomics analysis of cultures grown in complex media. Metabolomics 11, 198-209.

McCloskey, D., Young, J.D., Xu, S., Palsson, B.O., Feist, A.M., 2016a. MID Max: LC-MS/MS Method for Measuring the Precursor and Product Mass Isotopomer Distributions of Metabolic Intermediates and Cofactors for Metabolic Flux Analysis Applications. Anal. Chem. 88, 1362-1370.

McCloskey, D., Young, J.D., Xu, S., Palsson, B.O., Feist, A.M., 2016b. Modeling Method for Increased Precision and Scope of Directly Measurable Fluxes at a Genome-Scale. Anal. Chem. 88, 3844-3852.

Metcalf, W.W., Wanner, B.L., 1993. Evidence for a fourteen-gene, phnC to phnP locus for phosphonate metabolism in Escherichia coli. Gene 129, 27-32.

Miller, K., Dunsmore, C.J., Leeds, J.A., Patching, S.G., Sachdeva, M., Blake, K.L., Stubbings, W.J., Simmons, K.J., Henderson, P.J.F., De Los Angeles, J., Fishwick, C.W.G., Chopra, I., 2010. Benzothioxalone derivatives as novel inhibitors of UDP-N-acetylglucosamine 
enolpyruvyl transferases (MurA and MurZ). J. Antimicrob. Chemother. 65, 2566-2573.

Monje-Casas, F., Jurado, J., Prieto-Alamo, M.J., Holmgren, A., Pueyo, C., 2001. Expression analysis of the nrdHIEF operon from Escherichia coli. Conditions that trigger the transcript level in vivo. J. Biol. Chem. 276, 18031-18037.

Motiejūnaitè, R., Armalytè, J., Markuckas, A., Sužiedèlienè, E., 2007. Escherichia coli dinJ-yafQ genes act as a toxin-antitoxin module. FEMS Microbiol. Lett. 268, 112-119.

Murata-Kamiya, N., Kaji, H., Kasai, H., 1999. Deficient nucleotide excision repair increases base-pair substitutions but decreases TGGC frameshifts induced by methylglyoxal in Escherichia coli. Mutat. Res. 442, 19-28.

Murata-Kamiya, N., Kamiya, H., 2001. Methylglyoxal, an endogenous aldehyde, crosslinks DNA polymerase and the substrate DNA. Nucleic Acids Res. 29, 3433-3438.

Nagaraj, R.H., Shipanova, I.N., Faust, F.M., 1996. Protein cross-linking by the Maillard reaction. Isolation, characterization, and in vivo detection of a lysine-lysine cross-link derived from methylglyoxal. J. Biol. Chem. 271, 19338-19345.

Outten, F.W., Djaman, O., Storz, G., 2004. A suf operon requirement for Fe-S cluster assembly during iron starvation in Escherichia coli. Mol. Microbiol. 52, 861-872.

Ozyamak, E., de Almeida, C., de Moura, A.P.S., Miller, S., Booth, I.R., 2013. Integrated stress response of Escherichia coli to methylglyoxal: transcriptional readthrough from the nemRA operon enhances protection through increased expression of glyoxalase I. Mol. Microbiol. 88, 936-950.

Papoulis, A., al-Abed, Y., Bucala, R., 1995. Identification of N2-(1-carboxyethyl)guanine (CEG) as a guanine advanced glycosylation end product. Biochemistry 34, 648-655.

Plucain, J., Hindré, T., Le Gac, M., Tenaillon, O., Cruveiller, S., Médigue, C., Leiby, N., Harcombe, W.R., Marx, C.J., Lenski, R.E., Schneider, D., 2014. Epistasis and Allele Specificity in the Emergence of a Stable Polymorphism in Escherichia coli. Science 343, 1366-1369.

Plumbridge, J., 2009. An alternative route for recycling of $\mathrm{N}$-acetylglucosamine from peptidoglycan involves the $\mathrm{N}$-acetylglucosamine phosphotransferase system in Escherichia coli. J. Bacteriol. 191, 5641-5647.

Prysak, M.H., Mozdzierz, C.J., Cook, A.M., Zhu, L., Zhang, Y., Inouye, M., Woychik, N.A., 2009. Bacterial toxin YafQ is an endoribonuclease that associates with the ribosome and blocks translation elongation through sequence-specific and frame-dependent mRNA cleavage. Mol. Microbiol. 71, 1071-1087.

Ramisetty, B.C.M., Santhosh, R.S., 2017. Endoribonuclease type II toxin-antitoxin systems: functional or selfish? Microbiology 163, 931-939.

Ridderström, M., Mannervik, B., 2002. Glyoxalases I and II, in: Wiley Encyclopedia of Molecular Medicine. John Wiley \& Sons, Inc.

Roche, B., Huguenot, A., Barras, F., Py, B., 2015. The iron-binding CyaY and IscX proteins assist the ISC-catalyzed Fe-S biogenesis in Escherichia coli. Mol. Microbiol. 95, 605-623.

Saadat, D., Harrison, D.H.T., 1999. METHYLGLYOXAL SYNTHASE FROM ESCHERICHIA COLI. https://doi.org/10.2210/pdb1b93/pdb

Sandberg, T.E., Pedersen, M., LaCroix, R.A., Ebrahim, A., Bonde, M., Herrgard, M.J., Palsson, B.O., Sommer, M., Feist, A.M., 2014. Evolution of Escherichia coli to $42{ }^{\circ} \mathrm{C}$ and subsequent genetic engineering reveals adaptive mechanisms and novel mutations. Mol. Biol. Evol. 31, 2647-2662.

Shelley, S., 2007. A renewable route to propylene glycol. Chem. Eng. Prog. 103, 6-9.

Singh, R., Barden, A., Mori, T., Beilin, L., 2001. Advanced glycation end-products: a review. Diabetologia 44, 129-146.

Solá, M., Gomis-Rüth, F.X., Serrano, L., González, A., Coll, M., 1999. Three-dimensional crystal structure of the transcription factor PhoB receiver domain. J. Mol. Biol. 285, 675-687.

Tenaillon, O., Barrick, J.E., Ribeck, N., Deatherage, D.E., Blanchard, J.L., Dasgupta, A., Wu, 
G.C., Wielgoss, S., Cruveiller, S., Médigue, C., Schneider, D., Lenski, R.E., 2016. Tempo and mode of genome evolution in a 50,000-generation experiment. Nature 536, 165-170.

Thomas, M.C., 2011. Advanced glycation end products. Contrib. Nephrol. 170, 66-74.

Thornalley, P.J., 2003. Glyoxalase I--structure, function and a critical role in the enzymatic defence against glycation. Biochem. Soc. Trans. 31, 1343-1348.

Tokumoto, U., Nomura, S., Minami, Y., Mihara, H., Kato, S.-I., Kurihara, T., Esaki, N., Kanazawa, H., Matsubara, H., Takahashi, Y., 2002. Network of Protein-Protein Interactions among Iron-Sulfur Cluster Assembly Proteins in Escherichia coli1. J. Biochem. 131, 713719.

Torrents, E., 2014. Ribonucleotide reductases: essential enzymes for bacterial life. Front. Cell. Infect. Microbiol. 4, 52.

Umezawa, Y., Shimada, T., Kori, A., Yamada, K., Ishihama, A., 2008. The uncharacterized transcription factor $\mathrm{YdhM}$ is the regulator of the nemA gene, encoding $\mathrm{N}$-ethylmaleimide reductase. J. Bacteriol. 190, 5890-5897.

Vander Jagt, D.L., 1993. Glyoxalase II: molecular characteristics, kinetics and mechanism. Biochem. Soc. Trans. 21, 522-527.

Wanner, B.L., 1993. Gene regulation by phosphate in enteric bacteria. J. Cell. Biochem. 51, 4754.

Weber, J., Kayser, A., Rinas, U., 2005. Metabolic flux analysis of Escherichia coli in glucoselimited continuous culture. II. Dynamic response to famine and feast, activation of the methylglyoxal pathway and oscillatory behaviour. Microbiology 151, 707-716.

Xu, H.H., Real, L., Bailey, M.W., 2006. An array of Escherichia coli clones over-expressing essential proteins: a new strategy of identifying cellular targets of potent antibacterial compounds. Biochem. Biophys. Res. Commun. 349, 1250-1257.

Yamaguchi, Y., Park, J.-H., Inouye, M., 2009. MqsR, a crucial regulator for quorum sensing and biofilm formation, is a GCU-specific mRNA interferase in Escherichia coli. J. Biol. Chem. 284, 28746-28753.

Yang, C., Huang, T.-W., Wen, S.-Y., Chang, C.-Y., Tsai, S.-F., Wu, W.-F., Chang, C.-H., 2012. Genome-wide PhoB binding and gene expression profiles reveal the hierarchical gene regulatory network of phosphate starvation in Escherichia coli. PLoS One 7, e47314.

Fig. 1 Evolution of knockout (KO) strains from a pre-evolved (i.e., optimized) wild-type strain. A) Wild-type (wt) E. coli (MG1655 K-12) was previously evolved on glucose minimal media at $37^{\circ} \mathrm{C}$ (LaCroix et al., 2015). An isolate from the endpoint of the evolutionary experiment was selected as the starting strain for subsequent $\mathrm{KO}$ of tpiA and adaptive laboratory evolution (ALE). B) Adaptive laboratory evolution trajectories of the evolved knockout lineages. -Omics data collected included metabolomics, fluxomics, physiology, DNA resequencing, and transcriptomics. C) Triosephosphate isomerase (TPI) was disabled by the gene KO. TPI interconverts the triosephosphate dihydroxyacetone phosphate (dhap) to glyceraldehyde 3 phosphate (g3p). Additional metabolites shown for context include D-glucose (glc-D), glucose 6-phosphate (g6p), ribulose 5-phosphate (ru5p-D), methylglyoxal (mthgxl),

phosphoenolpyruvate (pep), pyruvate (pyr), DL-lactate (lac-DL), acetate (ac), alpha-ketoglutarate (akg), and succinate (succ). D) Growth rate and uptake and secretion rates of D-glucose, acetate, and DL-lactate for unevolved KO (UTpiA) and evolved KOs (eTpiA).

Fig. 2 The removal of triose phosphate isomerase (TPI) forced a bifurcation of flux in glycolysis through a pathway that involved the toxic intermediate methylglyoxal (mthgxl) (panels A-E). The bifurcated flux is rejoined by the lactate dehydrogenase (LDH) reaction. A) A network 
schematic of middle glycolysis. Shown along with removed TPI (shown in red) are the reactions catalyzed by fructose bisphosphate aldolase (FBA), glyceraldehyde 3-phosphate dehydrogenase (GAPD), and methylglyoxal synthase (MGSA). B) Flux levels of reactions derived from $\mathrm{C}^{13}$ labeling experiments near the gene knockout. C) Network schematic of lactate and pyruvate conversion. D) Regulatory schematic showing the feedback loop that upregulates the conversion of D/L-Lactate to pyruvate when elevated levels of intracellular lactate were sensed by the transcription factor IldR. E) Metabolite, expression, and measured flux levels involved in the regulatory feedback loop.

Fig. 3 Optimization of methylglyoxal detoxification pathway expression in tpiA lineages. Paths 1, 2 , and 3 were actively expressed in UTpiA, while path 4 was not. Through fixed mutations that affected gloA expression, path 4 was expressed in the eTpiA endpoints. A) Network diagram of the four methylglyoxal detox pathways in E. coli. B) Expression profiles of methylglyoxal detox pathway genes. Path 1: fucO and gldA are up regulated in uTpiA compared to all eTpiA strains; $y q h D$ and $d k g A$ are up regulated in uTpiA compared to Ref and are up regulated in most eTpiA strains compared to uTpiA; Path 2: AldA is up regulated in UTpiA compared to Ref and in most eTpiA strains compared to Ref, but down regulated in all eTpiA strains compared to uTpiA. Path 3: HchA is significantly elevated in uTpiA compared to all eTpiA strains and Ref; $y h b O$ is up regulated in eTpiA 3 compared to Ref. Path 4: GloA is up regulated in all eTpiA strains compared to Ref and uTpiA. Up/Down regulation p-value $<0.05$.

Fig. 4 Select mechanisms of methylglyoxal (mg or mthgxl) attack on cellular components. A) ball and stick diagram of methylglyoxal. Methylglyoxal contains two reactive carbonyls: an aldehyde and a ketone. Methylglyoxal exerts its toxicity through damage of cellular components with which it interacts. B) methylglyoxal cross-links single stranded RNA or DNA (shown) and/or peptides (shown in D) with other peptides between a RNA/DNA base and/or sulfur residues and lysine residues through formation of a imidazolysine group via the Maillard reaction (Broude and Budowsky, 1971; Krymkiewicz, 1973; Lo et al., 1994; Murata-Kamiya and Kamiya, 2001). C) methylglyoxal forms adducts with sulfur residues of enzymes directly that can inhibit their function (Kalapos, 1994; Ridderström and Mannervik, 2002). D) methylglyoxal participates in the formation of free radicals and reactive oxidative species (ROS) through glycation of amino acids (shown) or nucleotide bases (not shown) via Fenton's Reaction (Cadet et al., 2003; Cooke et al., 2003; Imlay and Linn, 1988; Kang, 2003). E) The generated ROS is then capable of inducing single stranded DNA or RNA (ssRNA/ssDNA) breaks. F) ROS attacks the ssRNA or ssDNA backbone generating 2-phosphoglycolate (2pgblyc) a deoxynucleotide and a nucleotide base. G) Metabolite concentrations of nucleobases, nucleotides, and mono-, di-, and tri- nucleotide phosphates. H) RNA expression profiles of select genes that respond to cellular stress and ROS.

Fig. 5 Mutations that affect the transcription factor NemR that allowed for expression of the gloA detox pathway(Ozyamak et al., 2013). A) Crystal structure of the nemR multimer (Gray et al., 2015). A mutation that alters NemR binding to the regulatory region are annotated in red. Chains $A, B, C$, and $D$ are highlighted in gold and grey; the regulatory region is highlighted in cyan; mutations are annotated in red. B) Reorientation and zoom in on two of the nemR multimer chains. C) Schematic of the nemRA-gloA operon (see Fig. 6 for expression profiles of the methylglyoxal detox pathways). NemR exerts negative feedback on the operon that is enhanced by increased levels of methylglyoxal (mthgxl) (Ozyamak et al., 2013; Umezawa et al., 
2008). gloA is co-expressed with nemR and nemA genes. D) Mutation frequency and expression profiles of nemR and nemR-associated genes. The lineages with mutations have significantly increased expression of the gloA detox pathway ( $p$-value $<0.05$ ).

Fig. 6 A host of mutations targeted elements of the phosphate starvation response regulon, including the PhoRB two component system (TCS) and the Pst phosphate ABC transporter. A) The PhoRB two component system (TCS) senses and responds to the absence of extracellular inorganic phosphate $(\mathrm{Pi})$, and is composed of a transmembrane sensory histidine kinase (PhoR) and transcription factor (PhoB). The PhoRB signalling processes are mediated by an $\mathrm{ABC}$ (ATP-dependent) transporter encoded by pstS, pstC, pstA, and pstB, that imports $\mathrm{Pi}$ as well as a chaperone-like inhibitory protein (PhoU) that physically interacts with the cytosolic portion of the Pst Pi ABC transporter (PstB) and PhoR. In the presence of extracellular Pi (Pi concentration approximately $>4 \mathrm{uM}$ ), the extracellular portion of the Pst Pi ABC transporter (PstS) is saturated. This leads to a signal transduction mediated by PhoU that leads to a conformational change of PhoR into an inactive state. Under conditions of phosphate limitation or in the absence of any of the Pst components, PhoR returns to its default active conformation, where it is able to phosphorylate and activate PhoB. B) Operon diagram for phoB. An 11 nucleotide deletion occurred at the PhoB TF binding site ( $s b c D-/$-phoB (DEL) $416288 \Delta 11 \mathrm{nt}$ ) in eTpiA03. C) A 1 nucleotide deletion at the 205 residue in phoB (phoB (DEL) 205 $\triangle 1 \mathrm{nt}$ ) in eTpiA03 in $61.3 \%$ of the population resulted in a frameshift that changed all amino acids from the site of the mutation to the end of the peptide. Interestingly, the peptide length was not changed. D) phoR encodes the transmembrane sensory histidine kinase component of the PhoRB TCS. Two, most likely deleterious mutations, targeted phoR. The phoR deletion at the 180 residue (phoR (DEL) $180 \Delta 1 \mathrm{nt}$ ) in eTpiA01 affected $53.2 \%$ of the population, and resulted in a frameshift that truncated the peptide at the 187 residue, cleaving the phosphohistidine and histidine kinase residue and domains, respectively. The phoR deletion at the 358 residue ( $p h o R$ (DEL) $358 \Delta 1 \mathrm{nt}$ ) in eTpiA04 resulted in a frameshift that truncated the peptide at the 368 residue, cleaving the histidine kinase domain, but leaving the phosphohistidine regulatory regions intact. E) Crystal structure of PhoB (Solá et al., 1999). PhoB is the response regulator (i.e., TF) component of the PhoRB TCS, and induces or represses operons of the Pho regulon. An SNP in phoB (phoB (SNP) A84P) occurred in eTpiA01 that affected $52.9 \%$ of the population. The mutation is located near the L105 residue that binds magnesium (mg2+), which may affect magnesium binding. The phoB deletion at the 39 residue (phoB (DEL) 39 $17 \mathrm{nt}$ ) in eTpiA03 resulted in a frameshift that truncated the peptide at the 89 residue. The phoB deletion at the 76 residue (phoB (DEL) 76 $55 \mathrm{nt}$ ) in eTpiA02 resulted in a frameshift that truncated the peptide at the 93 residue. Mutations are highlighted in red. The bound magnesium is shown in green. F) 
pst $A$ encodes a transmembrane component of the phosphate $\mathrm{ABC}$ transporter, which imports extracellular phosphate at the expense of ATP. A deleterious 774 nucleotide deletion in pstA (pstA (DEL) 1 $\Delta 774 \mathrm{nt}$ ) truncated the peptide to all but 38 amino acids. Loss of any of the Pst components has been shown to lead to a perpetual activation of the PhoRB TCS. G) Regulatory diagram of operons controlled by PhoB. H) Mutation frequency and expression of genes involved in phosphate starvation response regulon. Note that the majority of genes in the Phn and Pho operon are significantly elevated in UTpiA. See Table S4 for a comprehensive list of all significant changes in gene expression.

Fig. 7 Mutations that altered expression and function of nAcGam import and catabolic genes. A) Schematic of the nagBAC (catabolic enzymes) and nagE (PTS-specific component) operons. B) A network and mechanistic schematic of the nAcGam PTS Permease. N-acetyl glucosamine is imported from the periplasm and phosphorylated by EIIB (encoded by nagE. The phosphate is derived from the conversion of phosphoenolpyruvate (pep) to pyruvate (pyr) after a series of phosphorylation reactions along the PTS components EI, HPr, and ElIA. N-acetyl glucosamine phosphate (acgam6p) is then broken down to nitrate (nh4) and fructose 6-phosphate (f6p) by Nacetylglucosamine-6-phosphate deacetylase (AGDC) encoded by nagA and Glucosamine-6phosphate deaminase (G6PDA) encoded by nagB. C) Crystal structure of NagC. The majority of the nag $C$ mutations appeared to be deleterious. The nag $C$ mobile element insertion (MOB) mutation truncated the peptide from 409 AAs to 339 AAs. The nagC insertion (INS) mutation truncated the peptide from 409 to 96 AAs. The nagC SNP mutation results in a change of amino acids from $\mathrm{D}$ to $\mathrm{V}$. Mutations are highlighted in red, orange, and yellow. The DNA binding region is highlighted in cyan. The nagE mutations (not shown) occurred in the PTSEIIC type-1 complex formation region and resulted in amino acid changes (Table S8). The nagA SNP mutations (not shown) all resulted in amino acid changes (Table S8). The nagA MOB was most likely deleterious, and truncated the peptide from 382 to 168 AAs (Table S8). D) Mutation frequency and expression profiles of nagBAC and nagE genes. Note that nagE and nag $C$ were both mutated in eTpiA02/04, while lower level mutations in nagA occured in eTpiA01/03. While nagBAC and nagE are overexpressed in all eTpiA strains, eTpiA02/04 have significantly higher expression than eTpiA01/03.

Fig. 8 Mutations that increased expression of non-PTS glucose importers. Network and mechanistic schematic of the A) Glucose-specific PTS permease and B) passive and active glucose importers. Glucose is imported and phosphorylated from the periplasm into the cytosol by ElIB encoded by ptsG. C) Network schematic of operons regulated by GalR and GalS. D) Regulatory schematic of the GalP operon. The galP INS mutation occurred at the NagC binding site. Note that eTpiA01 had the highest level of expression of galP. E) crystal structure of the cytosolic portion of EIIA/B encoded by ptsG. The ptsG SNP introduced a stop codon that truncated the cytosolic portion of the protein including the PTS EIIB type-1 active site and phosphocysteine residue. The mutation is highlighted in red. The $\mathrm{cr}$ interaction domain is highlighted with a bounding box. The ptsG INS mutation (not shown) introduced an additional codon that shifted all residues by one in the PTS EIIB active site, which could have altered activity (Table S8). F) Crystal structure of GalR. Mutations are highlighted in red. The small molecule binding domain is highlighted in cyan. The DNA binding region is highlighted in 
magenta. G) Mutation frequency and expression levels of genes that compose the glucosespecific PTS as well as the D-galactose symporter and ABC transporter. Note that The Dgalactose symporter and $A B C$ transporter shown high affinity for glucose. PtsG is down regulated in UTpiA and all eTpiA strains compared to Ref. GalP is up regulated in eTpiA01 and 03 strains compared to UTpiA and Ref while UTpiA is down regulated in UTpiA compared to Ref. GalU is down regulated in UTpiA compared to Ref. GalS is up regulated in UTpiA and eTpiA strains compared to Ref. GalETKM operon genes are all up regulated in eTpiA01 and 03 strains. Up/Down regulation $p$-value $<0.05$.

Fig. 9 Mutations that affect pykF and murA activity. A) Network schematic of the PYK reactions. B) Crystal structure of PykF. Mutations are annotated in red. Substrate binding site residues are shown as ball and stick. The magnesium and potassium ion binding sites are highlighted in blue. The N6-acetyllysine residues are highlighted in cyan. The pykF MOB resulted in a truncated peptide that removed all features except for two potassium ion binding sites and a substrate binding residue. The pykFDEL was located at the interface between the dimers, and resulted in a frameshift that shifted the stop codon beyond the normal length of the peptide that most likely generated an aggregate peptide. The pykF SNP changed the 226 residue from $G$ to $D$, and was located in close proximity to the magnesium binding site residues. C) Network schematic of the UAGCVT reaction encoded by murA. D) Crystal structure of MurA (Eschenburg and Schonbrunn, 2004). The murA DEL resulted in a non-frameshift that shortened the peptide by one amino acid. Interestingly, the mutation changed the cysteine residue at position 115, which interacts with pep. The 115 residue in particular and murA as a whole has been actively investigated for implications in drug discovery (Bachelier et al., 2006; Barbosa et al., 2002; Baum et al., 2001; Couce et al., 2012; Desai and Miller, 2010; Dunsmore et al., 2008; Francisco et al., 2004; Kim et al., 1996; Miller et al., 2010; Xu et al., 2006). The mutated residue is highlighted in yellow. E) Mutation frequency, metabolite concentrations, expression, and metabolic flux derived from $\mathrm{C}^{13}$ labeling experiments of PYK and associated network components.

\section{Highlights:}

- tpiA Knockout-out in E. coli and adaptive laboratory evolution (ALE)

- Consequences of methylglyoxal toxicity and mechanisms for detoxification revealed

- Cross-talk between carbon starvation and inorganic phosphate signalling was uncovered

- Counter intuitive inactivation of PTS required to rebalance the flux map 


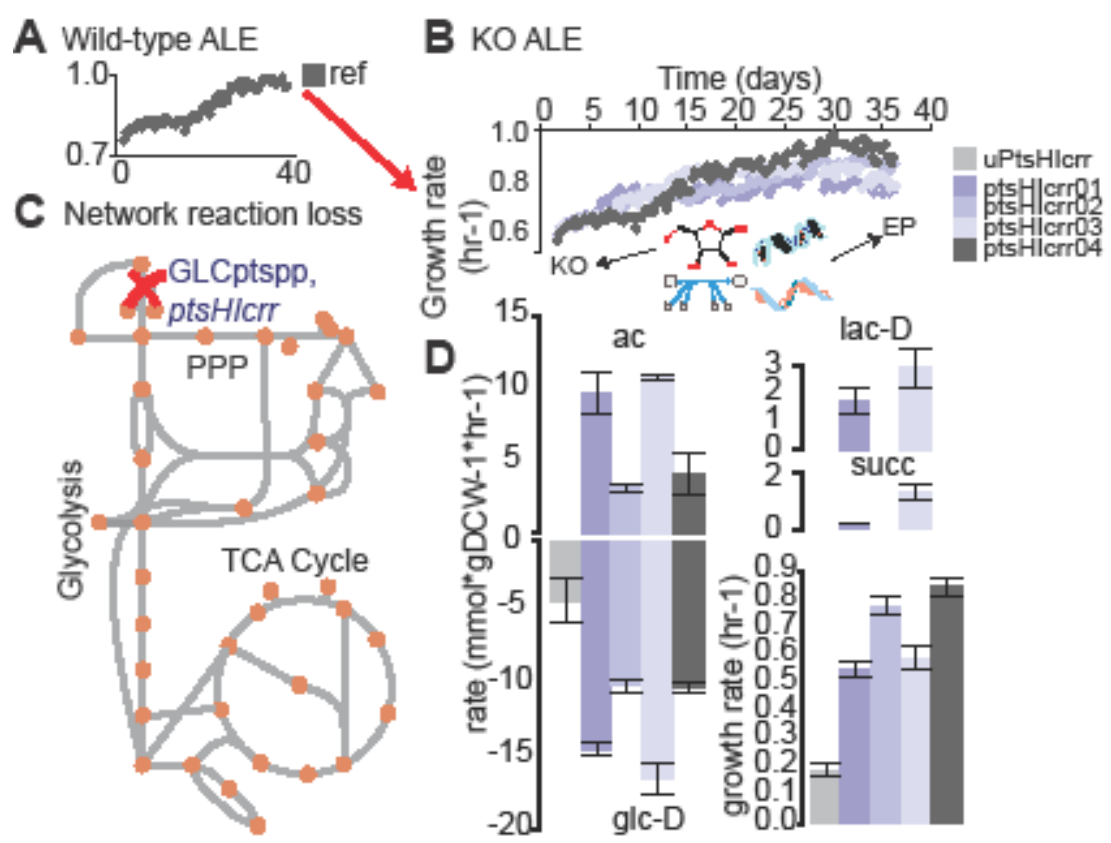



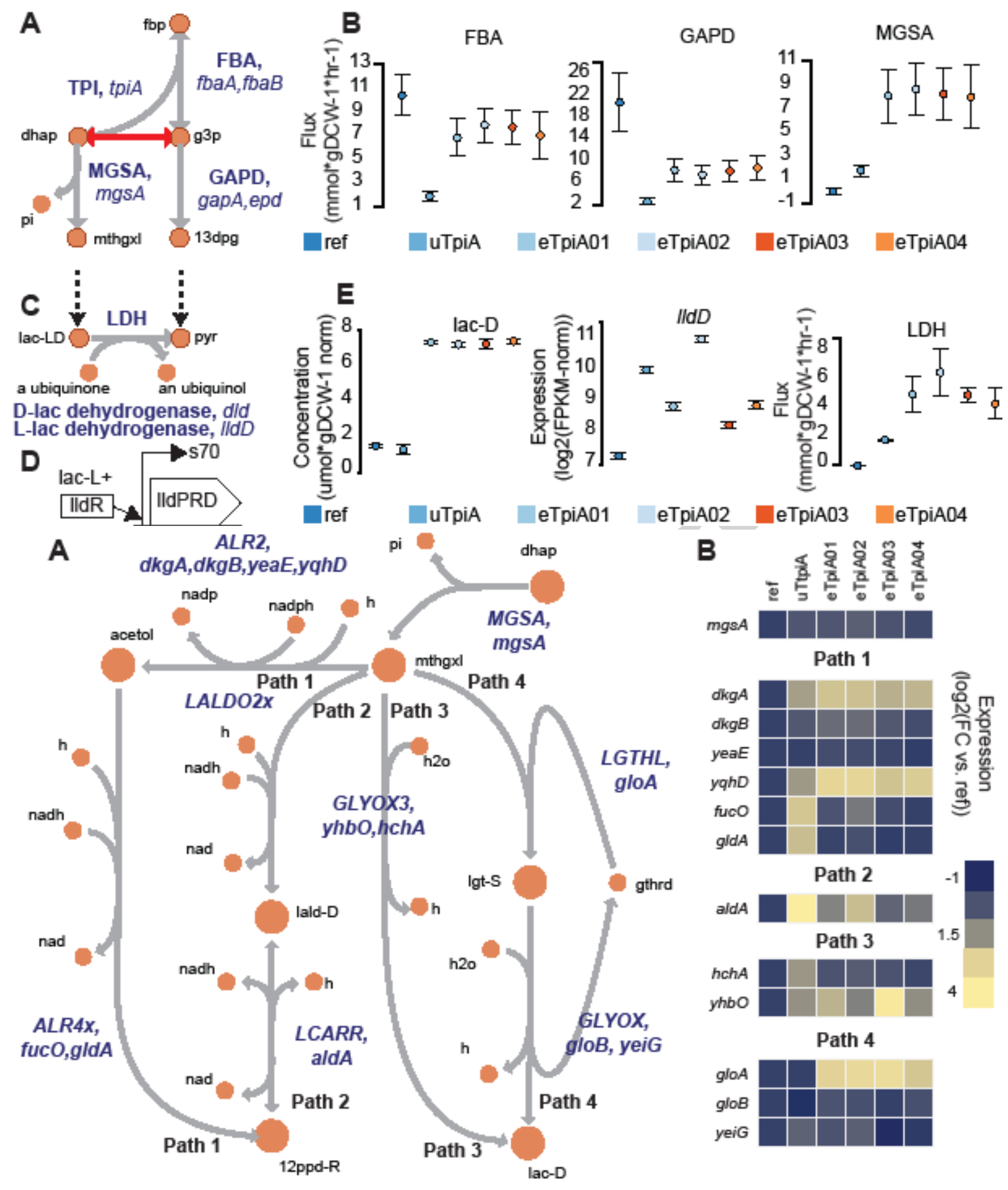

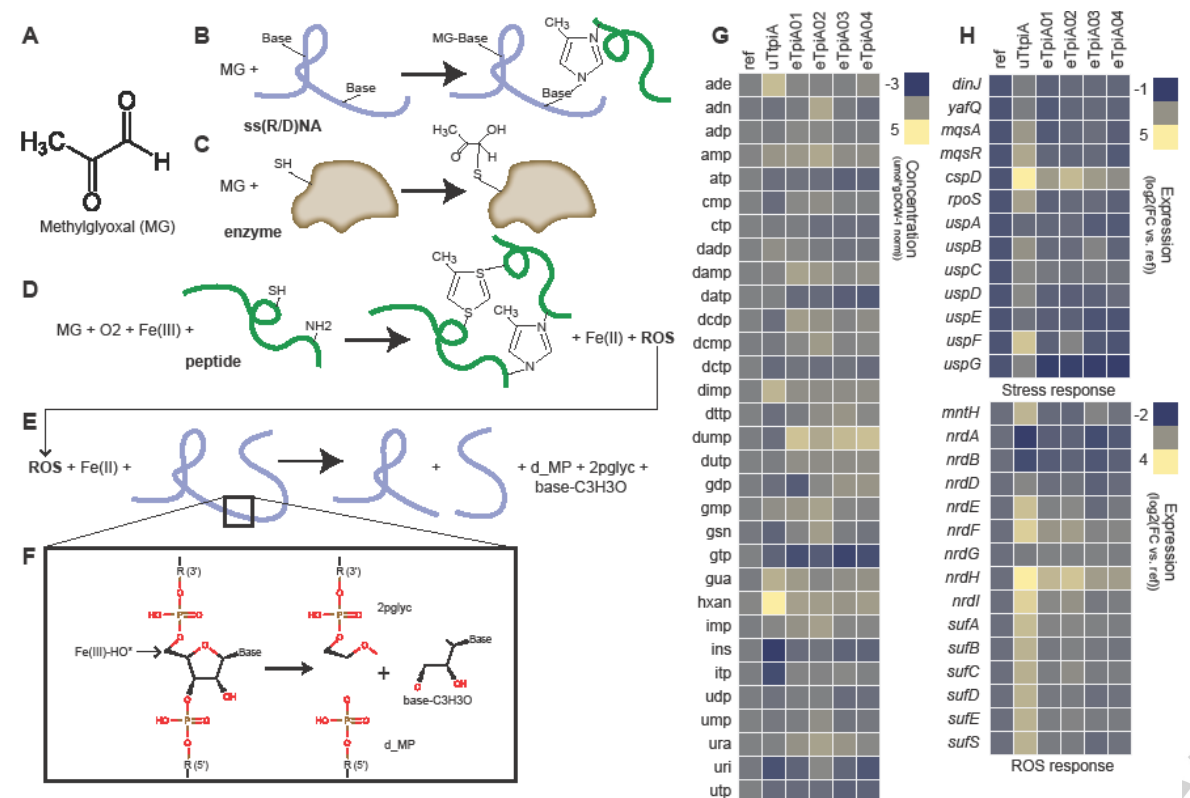

$$
\text { A nemR }
$$

Regulatory region

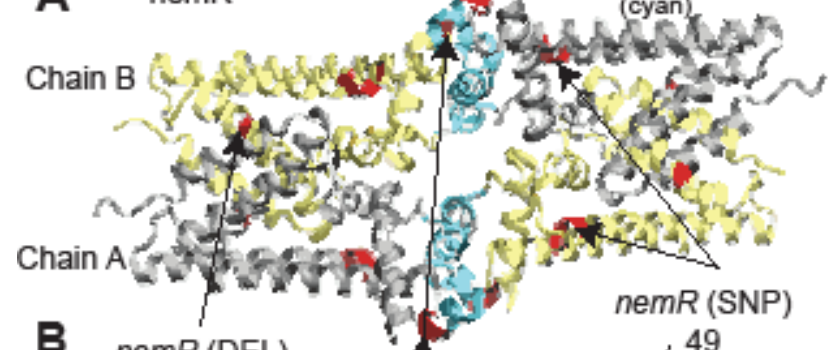

B $n e m R$ (DEL)

162

Chain C

NeTR (SNP)

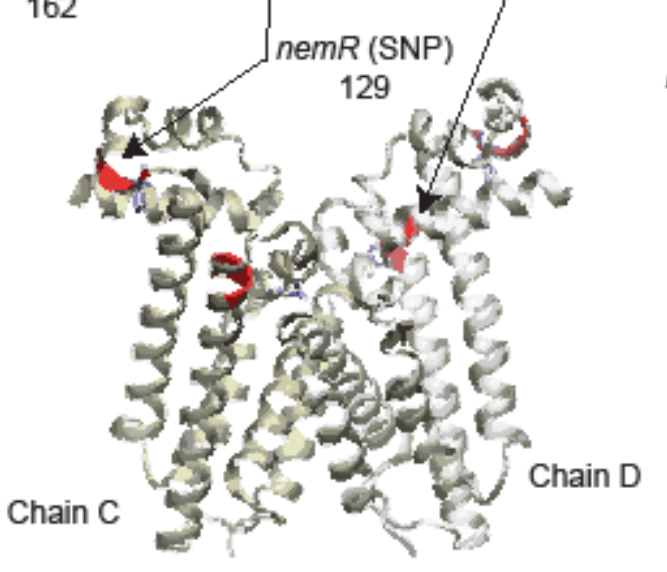

C

+ the

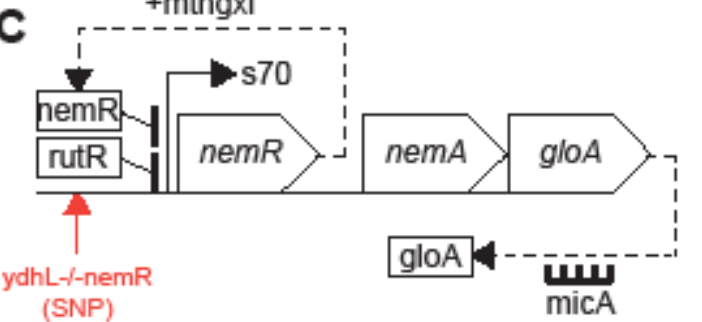

D

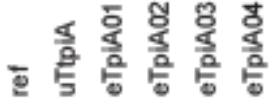
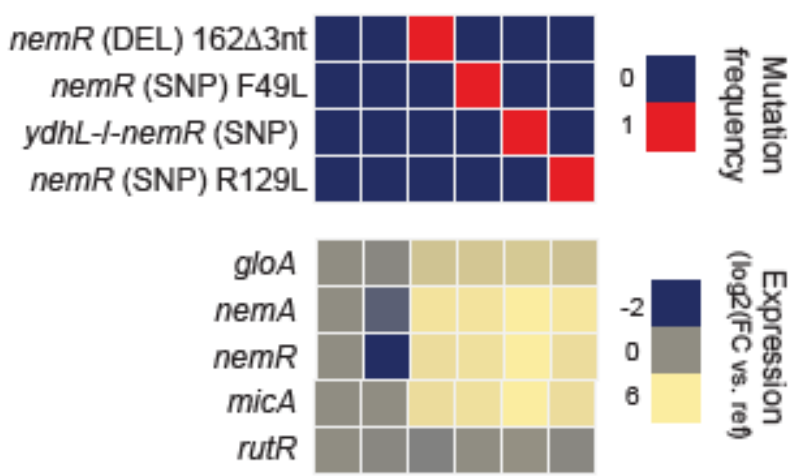


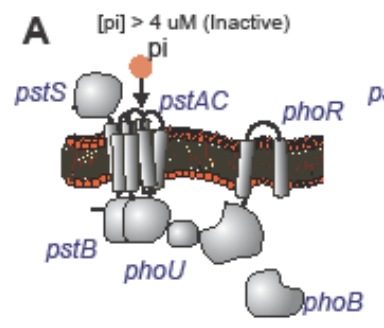

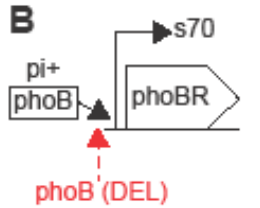

D
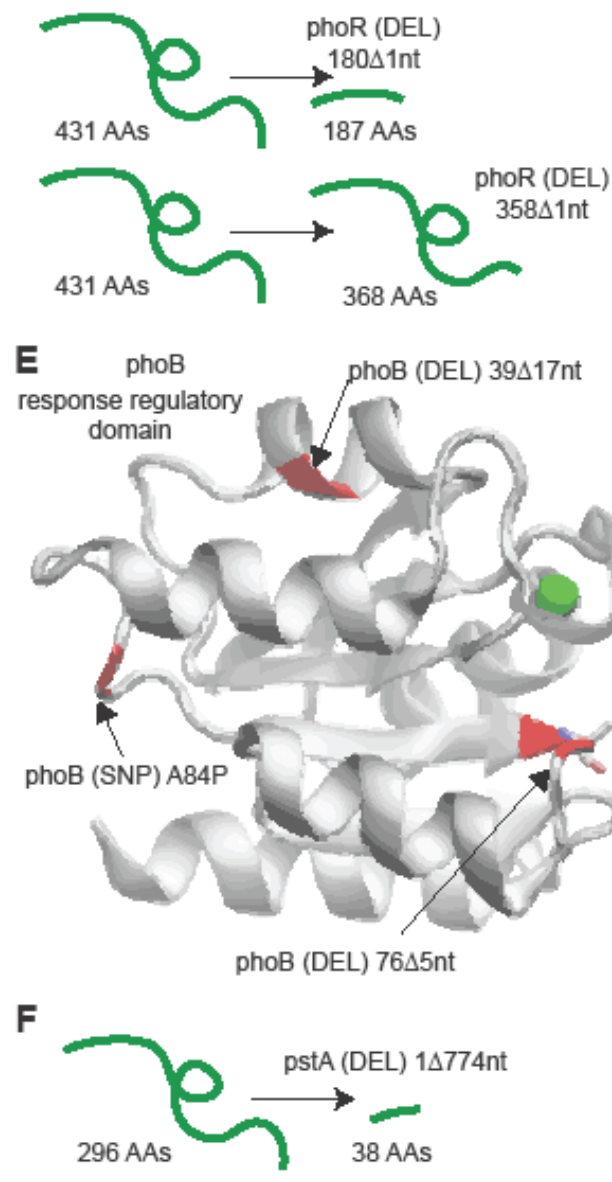

Irk [205] hwspagmtaw

crpcavqdivfqpaf [229] [pi] $<4$ uM (Active)

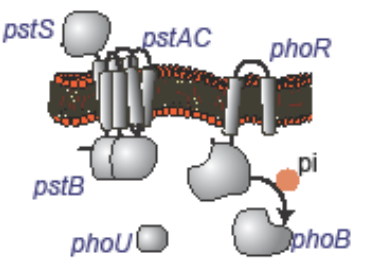

H

G

Transcription Factor Transcription unit cusA

.
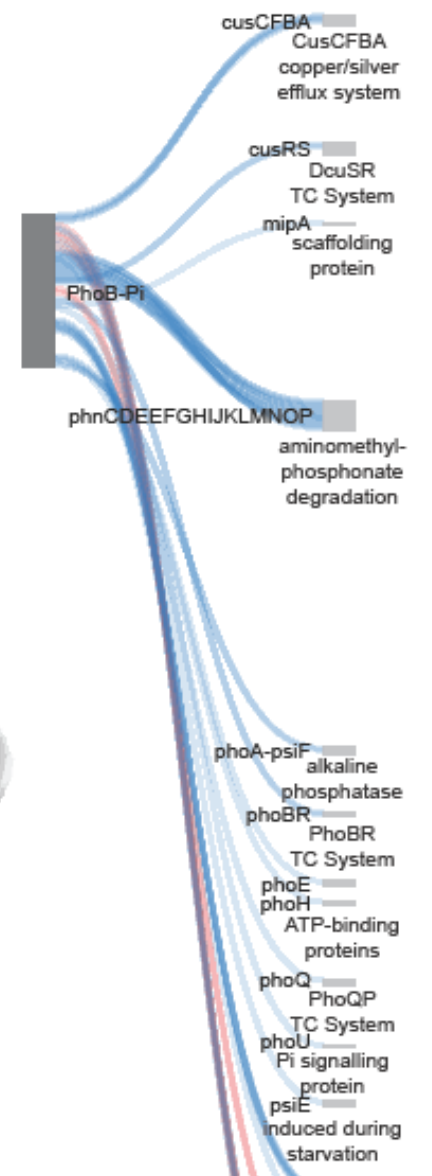

aminomethylphosphonate degradation $416288 \Delta 11 \mathrm{nt}$ phoB (SNP) A84P phoB (DEL) 39 $317 n t$ phoB (DEL) 76 $45 \mathrm{nt}$

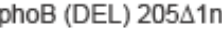
(DEL) $180 \Delta 1 \mathrm{nt}$ phoR (DEL) 358 $1 \mathrm{nt}$ pstA (DEL) $1 \Delta 774 \mathrm{nt}$

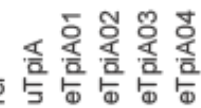
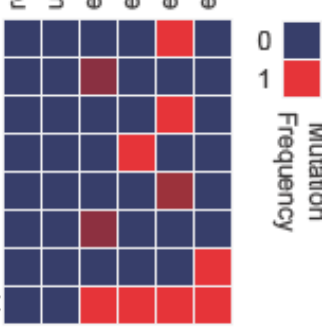

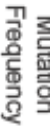

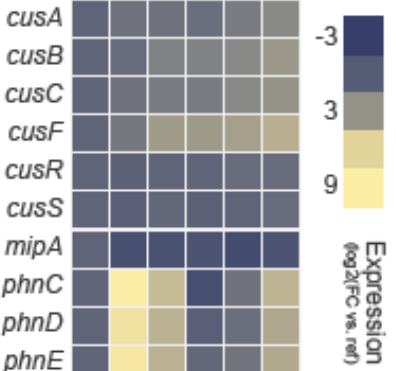


A

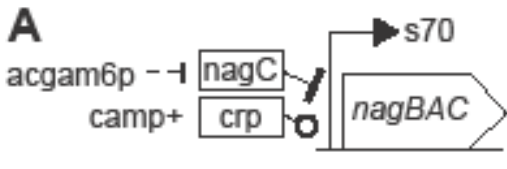

\section{B}

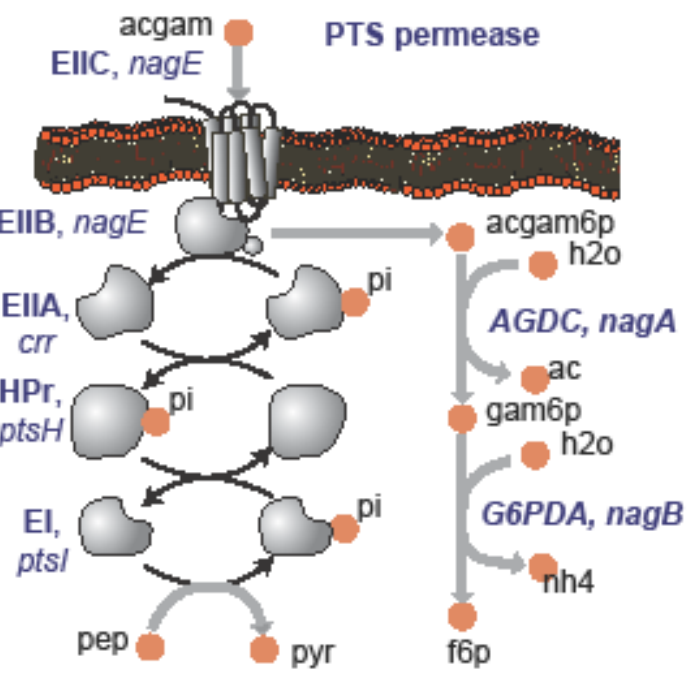

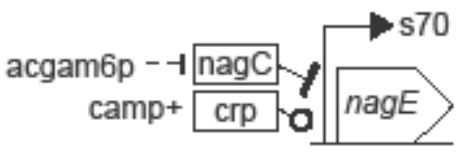

$\operatorname{nag} A$ (DEL) $141 \Delta 1 \mathrm{nt}$ nagA (SNP) P229R nagA (SNP) R149H nagA (SNP) H121P nagC (SNP) D66V nagC (INS) $73+1 \mathrm{nt}$ nagC (MOB) $333+\mid \mathrm{S} 1^{\star} 9$ nagE (SNP) $\mathrm{P} 22 \mathrm{Q}$ nagE (SNP) A47E
웅 웡 웅

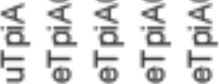
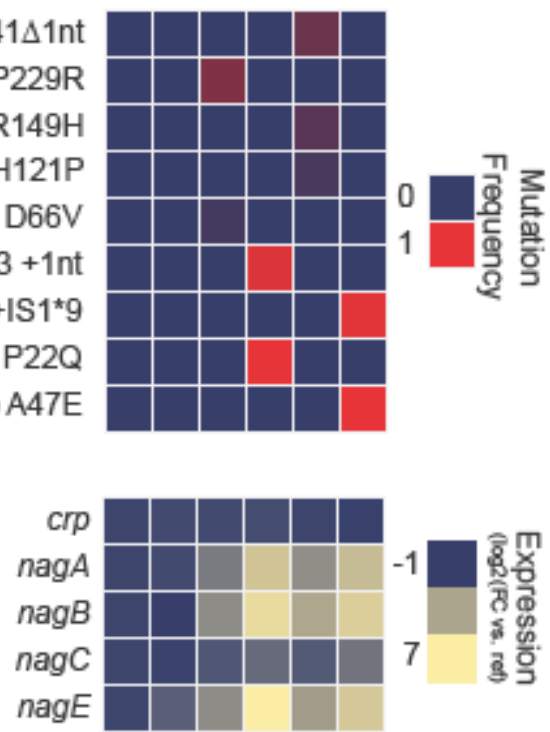

$\mathrm{H}-\mathrm{T}-\mathrm{H}$ DNA-binding region

nagC (MOB) $333+\mid S 1^{*} 9$

truncated remainder

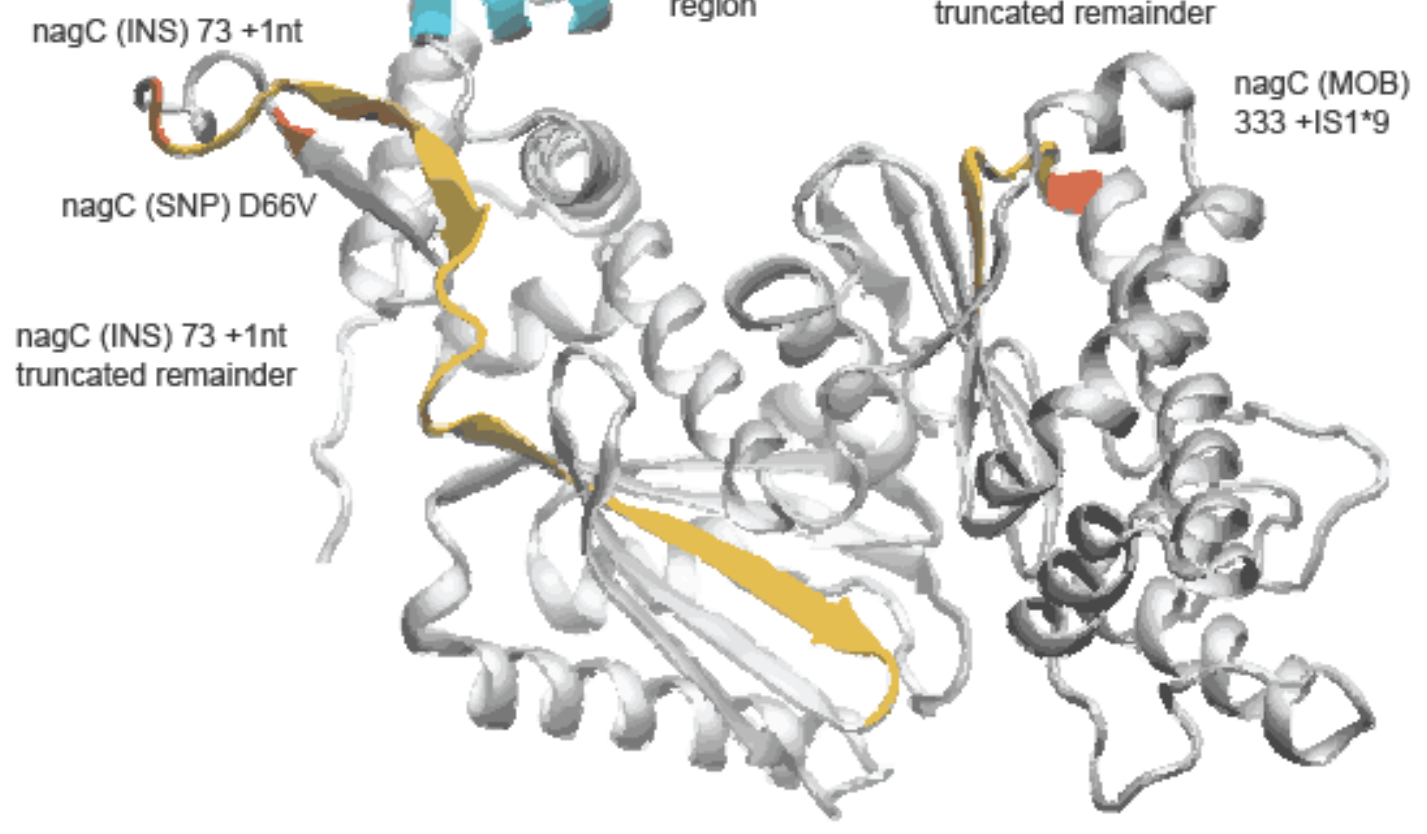




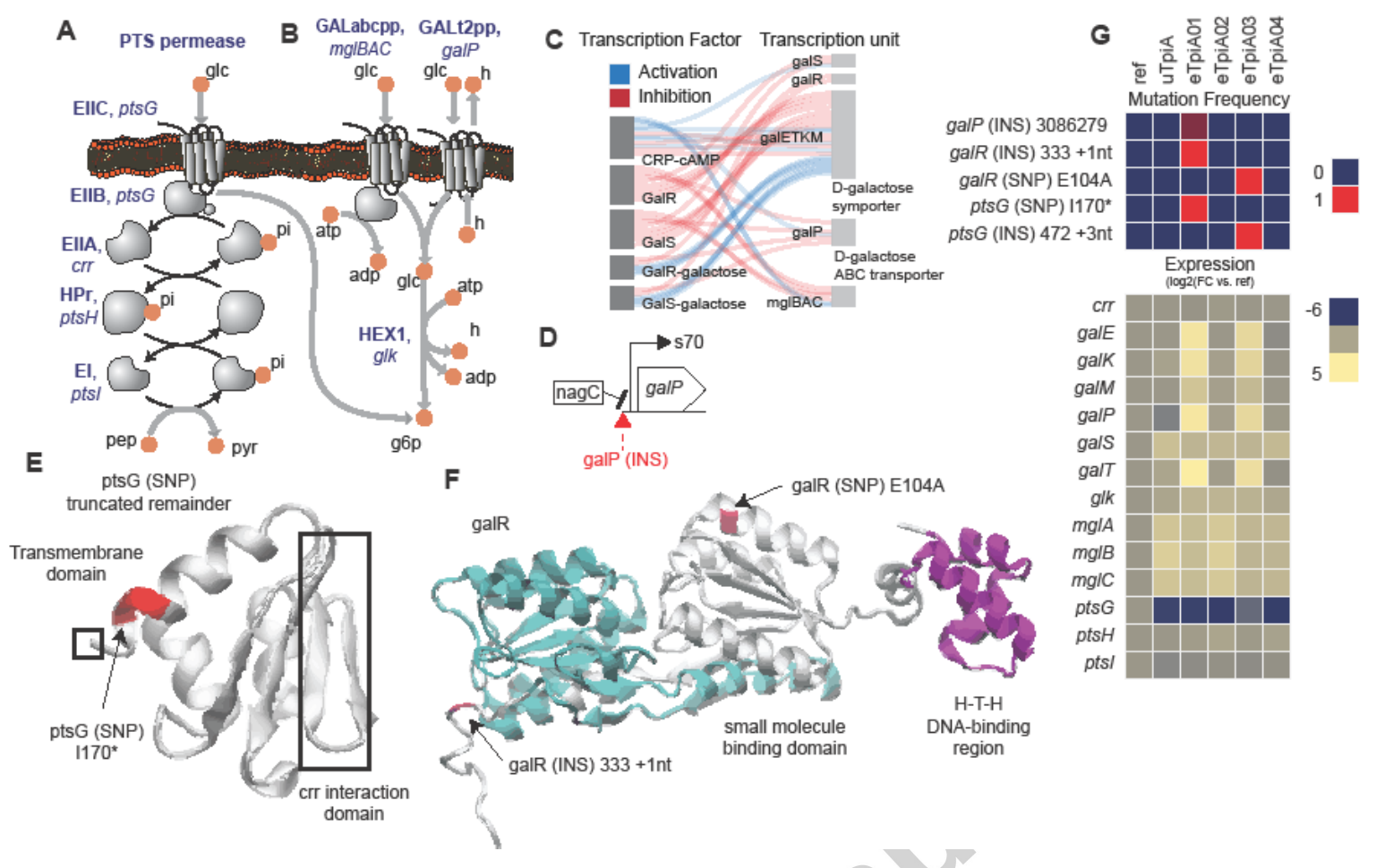




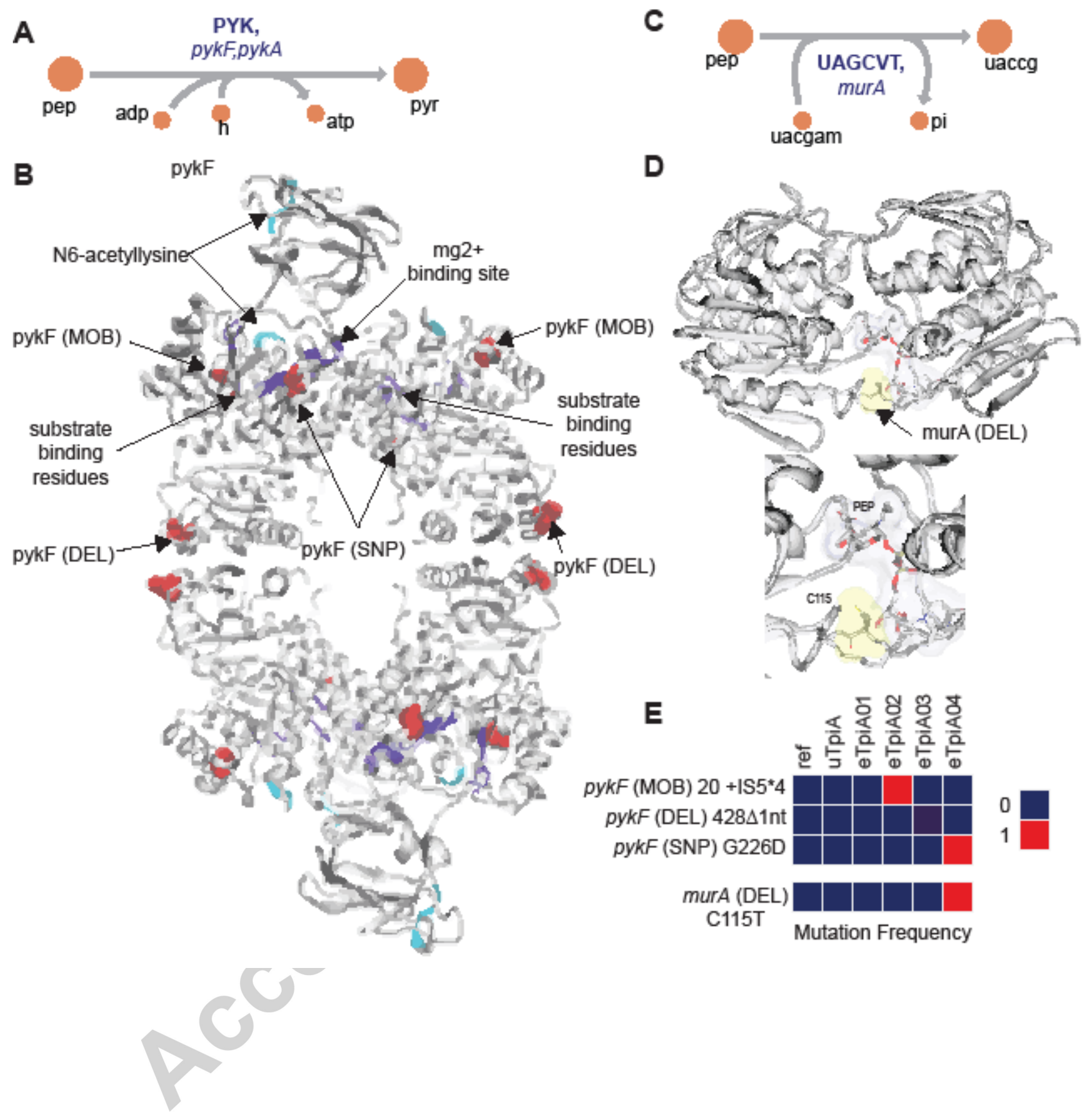

